\title{
Constraints on neutrino masses from Lyman-alpha forest power spectrum with BOSS and XQ-100
}

\section{Christophe Yèche, ${ }^{a, b}$ Nathalie Palanque-Delabrouille, ${ }^{a}$ Julien Baur, ${ }^{a}$ Hélion du Mas des Bourboux ${ }^{a}$}

\author{
${ }^{a}$ CEA, Centre de Saclay, IRFU/SPP, F-91191 Gif-sur-Yvette, France \\ ${ }^{b}$ Lawrence Berkeley National Laboratory, Berkeley, CA 94720, USA \\ E-mail: christophe.yeche@cea.fr, nathalie.palanque-delabrouille@cea.fr, \\ julien.baur@cea.fr,helion.du-mas-des-bourboux@cea.fr
}

\begin{abstract}
.
We present constraints on masses of active and sterile neutrinos in the context of the $\Lambda \mathrm{CDM} v$ and $\Lambda$ WDM models, respectively. We use the one-dimensional Ly $\alpha$-forest power spectrum from the Baryon Oscillation Spectroscopic Survey (BOSS) of the Sloan Digital Sky Survey (SDSS-III) measured by Palanque-Delabrouille et al. [1], and from the VLT/XSHOOTER legacy survey (XQ100). In this paper, we present our own measurement of the publicly released XQ-100 quasar spectra, focusing in particular on an improved determination of the spectrograph resolution that allows us to push to smaller scales than the public release and reach $k$-modes of $0.070 \mathrm{~s} \mathrm{~km}^{-1}$. We compare the obtained 1D Ly $\alpha$ flux power spectrum to the one measured by Irsic et al. [2] to $k$-modes of $0.057 \mathrm{~s} \mathrm{~km}^{-1}$.

Fitting Ly $\alpha$ data alone leads to cosmological parameters in excellent agreement with the values derived independently from Planck 2015 Cosmic Microwave Background (CMB) data. Combining BOSS and XQ-100 Ly $\alpha$ power spectra, we constrain the sum of neutrino masses to $\sum m_{v}<0.8 \mathrm{eV}$ ( $95 \%$ C.L.) including all identified sources of systematic uncertainties. With the addition of CMB data, this bound is tightened to $\sum m_{v}<0.14 \mathrm{eV}$ (95\% C.L.).

With their sensitivity to small scales, $\operatorname{Ly} \alpha$ data are ideal to constrain $\Lambda$ WDM models. Using XQ-100 alone, we issue lower bounds on pure dark matter particles: $m_{X} \gtrsim 2.08 \mathrm{keV}$ (95\% C.L.) for early decoupled thermal relics, and $m_{s} \gtrsim 10.2 \mathrm{keV}$ (95\% C.L.) for non-resonantly produced right-handed neutrinos. Combining the 1D Ly $\alpha$-forest power spectrum measured by BOSS and XQ100 , we improve the two bounds to $m_{X} \gtrsim 4.17 \mathrm{keV}$ and $m_{s} \gtrsim 25.0 \mathrm{keV}$ (95\% C.L.), slightly more constraining than what was achieved in Baur et al. 2015 [3] with BOSS data alone. The $3 \sigma$ bound shows a more significant improvement, increasing from $m_{X} \gtrsim 2.74 \mathrm{keV}$ for BOSS alone to $m_{X} \gtrsim$ $3.10 \mathrm{keV}$ for the combined BOSS+XQ-100 data set.

Finally, we include in our analysis the first two redshift bins $(z=4.2$ and $z=4.6)$ of the power spectrum measured by Viel et al. 2013 [4] with the high-resolution HIRES/MIKE spectrographs. The addition of HIRES/MIKE power spectrum allows us to further improve the two limits to $m_{X} \gtrsim$ $4.65 \mathrm{keV}$ and $m_{s} \gtrsim 28.8 \mathrm{keV}$ (95\% C.L.).
\end{abstract}




\section{Contents}

1 Introduction 1

$2 \quad X Q-100$ 1D power spectrum $\quad 4$

2.1 XQ-100 survey 4

2.2 The normalized transmitted flux fraction $\delta(\lambda) \quad 4$

2.3 Discussion of spectrograph resolution 5

2.4 Computation of the $P_{1 D}(k) \quad 6$

2.4.1 Method 6

2.4.2 Correction of the instrumental effects 7

$\begin{array}{lll}\text { 2.4.3 Correction of the metal absorption } & 7\end{array}$

2.4.4 Estimator of $P^{\mathrm{Ly} \alpha}(k) \quad 8$

3 Combining data 9

$\begin{array}{lll}3.1 & \text { Data } & 9\end{array}$

$\begin{array}{lll}3.1 .1 & \text { Ly } \alpha & 9\end{array}$

$\begin{array}{ll}\text { 3.1.2 Cosmic microwave background } & 11\end{array}$

$\begin{array}{ll}\text { 3.1.3 Baryon acoustic oscillations } & 11\end{array}$

$\begin{array}{lll}3.2 & \text { Simulations } & 11\end{array}$

$\begin{array}{llr}3.3 & \text { Methodology } & 12\end{array}$

4 Constraints on $\Lambda \mathrm{CDM} v \quad 13$

4.1 $\Lambda \mathrm{CDM} v \operatorname{cosmology}$ from Ly $\alpha$ data alone 13

4.2 $\Lambda \mathrm{CDM} v$ cosmology from Ly $\alpha$ data and other probes 13

5 Constraints on $\Lambda$ WDM $\quad 16$

5.1 $\Lambda$ WDM cosmology with XQ-100 16

5.2 Comparison with Irsic et al. Ly $\alpha$ power spectrum 18

$\begin{array}{ll}5.3 \text { Adding HIRES/MIKE power spectrum } & 20\end{array}$

6 Conclusion

\section{Introduction}

The flux power spectrum of the Lyman- $\alpha(\operatorname{Ly} \alpha)$ forest in quasar absorption spectra is a powerful tool to study clustering in the Universe at redshifts $\sim 2$ to 4 , on scales ranging from a few Mpc $[1,5,6]$ to hundreds of Mpc [7-9]. Compared to a model derived from a set of dedicated hydrodynamical simulations, the $\operatorname{Ly} \alpha$-flux power spectrum can provide valuable information on the formation of structures and their evolution. In particular, by probing scales down to a few Mpc, the 1D flux power spectrum is sensitive to neutrino masses through the suppression of power on small scales that neutrinos induce. Being relativistic until late in the history of the Universe, neutrinos free-stream out of gravitational potentials and therefore damp small-scale density fluctuations for at least two reasons: by the absence of neutrino perturbations in the total matter power spectrum, but even more so by slowing down the growth rate of baryons and CDM perturbations since neutrinos contribute to the background density, and thus to the expansion rate, but not to the clustering. The overall effect of massive neutrinos is a 
step-like suppression of power by a factor of order $1-8\left(\Omega_{v} / \Omega_{m}\right)$, where the present neutrino energy density relative to the critical density is given by $\Omega_{v}=\sum m_{v} /\left(93.14 h^{2} \mathrm{eV}^{2}\right)[10,11]$.

Cosmic Microwave Background (CMB) data can also constrain $\sum m_{v}$. In the standard thermal history of the Universe, massless neutrinos have a temperature $T_{v}=0.18 \mathrm{eV}$ at the epoch of last scattering, corresponding to an average momentum $\langle p\rangle=3.15 T_{v}=0.57 \mathrm{eV}$. This temperature sets the range of masses for which neutrinos start to have an appreciable effect on the CMB power spectrum to $\sum m_{v}>3 \times 0.57=1.7 \mathrm{eV}$ (for three active neutrino species). Below this mass, the neutrinos are still relativistic at recombination and have no direct impact on the primary CMB anisotropies. The effect of neutrino mass on the CMB then only appears at the level of secondary anisotropies, through the integrated Sachs-Wolf effect or the weak lensing by foreground gravitational structures. Using a measurement of these effects, the latest limit set on $\sum m_{v}$ by the Planck team from CMB data alone is at the level of $0.7 \mathrm{eV}$ [12].

Despite a clear suppression in the power spectrum, Ly $\alpha$ data alone also exhibit a sensitivity to $\sum m_{v}$ at the level of about $1 \mathrm{eV}$ only, due to the fact that the scales probed by Ly $\alpha$ forests are in a region of scales where the ratio of the power spectra for massive to massless neutrinos is quite flat. However, a tight constraint on $\sum m_{v}$ can be obtained by combining Ly $\alpha$ with CMB data: while Ly $\alpha$ data probe the suppressed power spectrum, CMB data on the other hand probe the power spectrum on scales large enough to be unaffected by the free-streaming of neutrinos. The combination of data from small and large scales therefore provides a direct measure of the suppression, and thus on $\sum m_{v}$. In practice, Ly $\alpha$ measures the power spectrum level, defined by $\sigma_{8}$ and $\Omega_{m}$, CMB provides the correlations between these parameters and $\sum m_{v}$, and the joint use of these two probes significantly improves the constraint on $\sum m_{v}$ compared to what either probe alone can achieve.

By interfering with the gravitational collapse of structures while they are relativistic, warm dark matter particles also significantly affect the matter power spectrum and can be studied in a similar way as active neutrinos. In the case of $\Lambda \mathrm{WDM}$ models where all the dark matter is assumed to be in the form of WDM particles with masses of a few $\mathrm{keV}$, the linear 3D matter power spectrum shows a complete cut-off on scales above $k \sim 2 \pi / r \sim 10 h \mathrm{Mpc}^{-1}$ [13-15]. This free-streaming-induced cut-off translates into a gradual suppression on the 1D flux power spectrum that falls within the range of scales probed by the $\operatorname{Ly} \alpha$ forest of distant high redshift quasars. Ly $\alpha$ forest data therefore again provide an ideal tool to study keV-range WDM and yield lower bounds on the mass of early decoupled thermal relics or right-handed sterile neutrinos, for instance.

In this paper, we exploit Ly $\alpha$ data from three surveys. For the Baryon Oscillation Spectroscopic Survey (BOSS) of the Sloan Digital Sky Survey (SDSS-III), we use the 1D Ly $\alpha$ flux power spectrum measured by [1] with the quasar data of the DR9 release. For VLT/XSHOOTER legacy survey XQ100 [16], we directly compute our own $1 \mathrm{D}$ power spectrum from the publicly released XQ-100 quasar spectra. A comparison with the power spectrum measured by [2] is discussed in this paper. Finally we add to the BOSS+XQ-100 data set, the power spectrum measured for two redshift bins $(z=4.2$ and $z=4.6$ ) with the high-resolution HIRES/MIKE spectrographs and described in [4].

The simulations we use to interpret the $\operatorname{Ly} \alpha$ data come from a grid of 36 hydrodynamical simulations having a resolution equivalent to $3 \times 3072^{3}$ particles in a $\left(100 h^{-1} \mathrm{Mpc}\right)^{3}$ box $[17,18]$. We use these simulations to predict the flux power spectrum and constrain cosmology, the sum of the neutrino masses $\sum m_{v}$ and the mass of WDM particles.

The layout of the paper is as follows. Sec. 2 presents the measurement of the Ly $\alpha$ forest power spectrum from the VLT/XSHOOTER legacy survey XQ-100 data. We explain the various steps of the analysis and detail the non-standard issues that we addressed with specific care, such as the determination of the spectrograph resolution in the present case where the seeing of the observations is smaller than the slit size. Sec. 3 gives a brief summary of the Ly $\alpha$ forest, CMB and Baryon 
Acoustic Oscillation (BAO) data sets that we use in this work. We also introduce the hydrodynamical simulations from which we build our likelihood. The main objective of Sec. 4.1 is to present the cosmological constraints that can be achieved from Ly $\alpha$ data alone (BOSS and XQ-100). The base model we consider is a flat $\Lambda \mathrm{CDM}$ cosmology with massive neutrinos, thereafter referred to as the base $\Lambda \mathrm{CDM} v$ cosmology. In Sec. 4.2, we include additional data, namely several configurations of CMB and BAO measurements. We present the results obtained on the parameters of our base $\Lambda \mathrm{CDM} v \operatorname{cosmology}$ using various combinations of the data sets. Finally, in Sec. 5, we discuss our results in the framework of $\Lambda \mathrm{WDM}$ models and give lower limits on the mass of thermal relics and non-resonantly produced sterile neutrino.

This paper refers extensively to the earlier papers that reported constraints on cosmological parameters and the mass of active neutrinos [19] or constraints on WDM and the mass of sterile neutrinos [3] using Ly $\alpha$ data from the SDSS-III/BOSS survey. For the sake of simplicity, we will henceforth refer to [19] as PY15 and to [3] as BP16. We also refer the reader to [17] for a detailed description of the grid of hydrodynamical simulations used in this work, and to [18] for the implementation of neutrinos and their impact on the 1D flux power spectrum. Definitions of the most relevant symbols used in this paper can be found in Tables 1 and 2.

Table 1: Definition of astrophysical parameters

\begin{tabular}{|c|c|}
\hline Parameter & Definition \\
\hline$\delta=\rho /\langle\rho\rangle$ & Normalized baryonic density $\rho$ of IGM \\
\hline$T \ldots \ldots$ & Temperature of IGM modeled by $T=T_{0} \cdot \delta^{\gamma-1}$ \\
\hline$T_{0} \ldots \ldots \ldots \ldots$ & Normalization temperature of IGM at $z=3$ \\
\hline$\gamma \ldots \ldots \ldots \ldots$ & Logarithmic slope of $\delta$ dependence of IGM temperature at $z=3$ \\
\hline$\eta^{T_{0}}$ & Logarithmic slope of redshift dependence of $T_{0}$ (different for $z<$ or $\left.>3\right)$ \\
\hline$\eta^{\gamma}$ & Logarithmic slope of redshift dependence of $\gamma$ \\
\hline$A^{\tau}$ & Effective optical depth of $\operatorname{Ly} \alpha$ absorption at $z=3$ \\
\hline$\eta^{\tau}$ & Logarithmic slope of redshift dependence of $A^{\tau}$ \\
\hline$f_{\text {Si III }} \ldots \ldots \ldots \ldots$ & Fraction of Si III absorption relative to $\operatorname{Ly} \alpha$ absorption \\
\hline$f_{\text {Si II }} \ldots \ldots \ldots \ldots$ & Fraction of Si II absorption relative to Ly $\alpha$ absorption \\
\hline
\end{tabular}

Table 2: Definition of cosmological parameters

\begin{tabular}{|c|c|}
\hline Parameter & Definition \\
\hline$\Omega_{m} \ldots$ & Matter fraction today (compared to critical density) \\
\hline$H_{0}$ & Expansion rate today in $\mathrm{km} \mathrm{s}^{-1} \mathrm{Mpc}^{-1}$ \\
\hline$\sigma_{8} \ldots$ & RMS matter fluctuation amplitude today in linear theory \\
\hline$\ldots \ldots \ldots$ & Optical depth to reionization \\
\hline$\ldots \ldots \ldots \ldots$ & Redshift where reionization fraction is $50 \%$ \\
\hline$n_{s} \ldots \ldots \ldots \ldots \ldots \ldots$ & Scalar spectral index \\
\hline$\sum m_{v} \ldots \ldots \ldots \ldots \ldots$ & Sum of neutrino masses in eV \\
\hline$m_{X} \ldots \ldots \ldots \ldots \ldots \ldots$ & Mass of thermal relics in $\mathrm{keV}$ \\
\hline$m_{s} \ldots \ldots \ldots \ldots \ldots \ldots$ & Mass of non-resonantly produced sterile neutrino in $\mathrm{keV}$ \\
\hline
\end{tabular}




\section{XQ-100 1D power spectrum}

In this section we present our measurement of the one-dimensional Ly $\alpha$ forest power spectrum from the quasars of the XQ-100 project. After a brief description of the data, we explain the analysis of the spectra, building upon the method developed for BOSS and described in [1]. Finally, we compare our results to the measurement of the power spectrum from the same data set by Irsic et al. [2].

\subsection{XQ-100 survey}

XQ-100, "Quasars and their absorption lines: a legacy survey of the high-redshift universe with VLT/XSHOOTER", is one of the large programmes of the European Southern Observatory [16]. The survey consists of a homogeneous and high-quality sample of 100 echelle spectra of quasars at redshifts $z \simeq 3.5-4.5$. The quasars were observed with full spectral coverage from 3150 to $25000 \AA$, at a resolving power ranging from $\sim 4000$ to 7000 , depending on wavelength.

We use both the XQ-100 raw data and the XQ-100 Science Data Products (SDP) released at http://archive.eso.org/eso/eso_archive_main.html and http://archive.eso.org/ $\mathrm{wdb} / \mathrm{wdb} / \mathrm{adp} /$ phase3_main/form, respectively. The main part of our work relies on the SDP spectra; the raw spectra are only exploited for the study and validation of the spectrograph resolution, see Sec. 2.3.

The region of interest for this study, the Ly $\alpha$ forest, is covered by the UVB ( $3150-5600 \AA)$ and VIS (5 400 - $10200 \AA$ ) spectroscopic arms of XSHOOTER. In the Ly $\alpha$ forest, the signal-to-noise ratio per pixel varies from 5 to 60 , with an average of $\sim 25$. The spectral resolution depends on the arm. It is respectively $12 \mathrm{~km} \mathrm{~s}^{-1}$ and $18 \mathrm{~km} \mathrm{~s}^{-1}$ on average for the VIS and UVB arms respectively.

For comparison, about 700 BOSS quasars in the same $\mathrm{HI}$ absorption region $(z \simeq 3.0-4.2)$ were analyzed in [1]. BOSS quasars exhibit a signal-to-noise ratio typically 10 to 20 times lower and a spectral resolution 3 to 5 times worse than XQ-100 quasars. As a consequence, BOSS data allow us to compute the power spectrum to scales at most of $0.02 \mathrm{~s} \mathrm{~km}^{-1}$, while XQ-100 data allow us to reach much smaller scales, corresponding to $0.07 \mathrm{~s} \mathrm{~km}^{-1}$ for the VIS arm of XSHOOTER that has the better resolution.

\subsection{The normalized transmitted flux fraction $\delta(\lambda)$}

In Fig. 1, we show a typical XQ-100 spectrum (left plot) and the average quasar spectrum (right plot) obtained by averaging all XQ-100 quasar spectra split into 3 redshift bins. Broad quasar emission lines are clearly visible, such as Ly $\beta(1026 \AA)$, Ly $\alpha(1216 \AA), N_{\text {v }}(1240 \AA), S i$ Iv $(1400 \AA)$ and C IV $(1549 \AA)$, where all wavelengths are expressed in rest frame. Absorption by Ly $\alpha$ absorbers along a quasar line of sight appears blueward of the quasar Ly $\alpha$ emission peak, with more absorption (and hence less transmitted flux) at high redshift.

We define the Ly $\alpha$ forest by the range $1040<\lambda_{\mathrm{RF}}<1200 \AA$, thus $\sim 4000 \mathrm{~km} \mathrm{~s}^{-1}$ away from the quasar $\operatorname{Ly} \beta$ and $\operatorname{Ly} \alpha$ emission peaks to avoid contamination of the power spectrum by astrophysical effects in the vicinity of the quasar. The Ly $\alpha$ forest spans a redshift range $\Delta z \sim 0.65$ for a quasar at a redshift $z_{\mathrm{qso}}=4$. In order to improve the redshift resolution, we split the Ly $\alpha$ forest into three consecutive and non-overlapping sub-regions of equal length, hereafter called ' $z$-sectors'. The splitting of each spectrum is done in such a way as to ensure that a ' $z$-sector' lies on a single spectroscopic arm (either VIS or UVB), and to avoid the parts of the forest containing Damped Lyman Alpha (DLA) or Lyman limit (LLS) systems detected by [20].

The largest possible mode is determined by the Nyquist-Shannon limit at $k_{\text {Nyquist }}=\pi / \Delta v$. With a pixel size $\Delta v=c \Delta \lambda / \lambda=20 \mathrm{~km} \mathrm{~s}^{-1}$ and $11 \mathrm{~km} \mathrm{~s}^{-1}$ for the UVB and VIS arms, the largest mode is, respectively, $k_{\text {Nyquist }}=0.16 \mathrm{~s} \mathrm{~km}^{-1}$ and $0.29 \mathrm{~s} \mathrm{~km}^{-1}$. We limit the analysis, however, to $k_{\max }=$ 

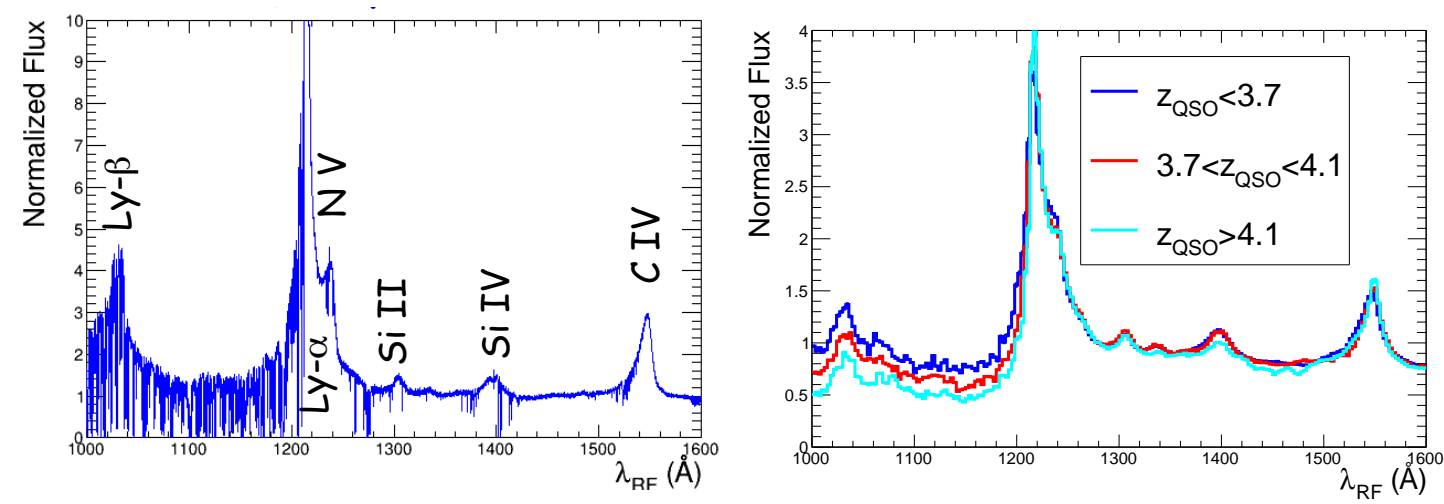

Figure 1: Left: Example of a typical quasar spectrum observed by XQ-100. Right: Average quasar spectra in three redshift bins. All spectra are normalized at $\lambda=1280 \AA$.

$0.05-0.07 \mathrm{~s} \mathrm{~km}^{-1}$, depending on the absorption redshift, because of the large window function correction, at the largest $k$-modes, mostly due to the spectrograph resolution.

Sky lines affect the data quality by increasing the pixel noise. We identify major sky lines (such as lines at 5577, 5890,6300,6364, and $6864 \AA$ ) and we replace the flux of each pixel impacted by a sky line by the average value of the flux over the rest of the forest. This procedure introduces a small $k$-dependent bias in the resulting power spectrum, which is negligible in this analysis due to the small number of sky lines in this wavelength region and the good spectrograph resolution.

The normalized transmitted flux fraction $\delta(\lambda)$ is estimated from the pixel flux $f(\lambda)$ by:

$$
\delta(\lambda)=\frac{f(\lambda)}{f_{\mathrm{qso}}^{1280} C_{q}\left(\lambda, z_{\mathrm{qso}}\right) \bar{F}\left(z_{\mathrm{Ly} \alpha}\right)}-1,
$$

where $f_{\text {qso }}^{1280}$ is a normalization equal to the mean flux in a $20 \AA$ window centered on $\lambda_{R F}=1280 \AA$ where $\lambda_{R F}=\lambda /\left(1+z_{\mathrm{qso}}\right), C_{q}\left(\lambda, z_{\mathrm{qso}}\right)$ is the normalized unabsorbed flux (the mean quasar 'continuum') and $\bar{F}\left(z_{\mathrm{Ly} \alpha}\right)$ is the mean transmitted flux fraction at the $\mathrm{H}_{\mathrm{I}}$ absorber redshift. Pixels affected by sky line emission are not included when computing the normalization. Since the mean quasar continuum is flat in the normalization region, the rejection of a few pixels does not bias the mean pixel value. The product $C_{q}\left(\lambda, z_{\mathrm{qso}}\right) \bar{F}\left(z_{\mathrm{Ly} \alpha}\right)$ is assumed to be universal for all quasars at redshift $z_{\mathrm{qso}}$ and is computed by stacking appropriately-normalized quasar spectra $f / f_{\mathrm{qso}}^{1280}$, thus averaging out the fluctuating Ly $\alpha$ absorption. The product $f_{\mathrm{qso}}^{1280} C_{q}\left(\lambda, z_{\mathrm{qso}}\right) \bar{F}\left(z_{\mathrm{Ly} \alpha}\right)$ represents the mean expected flux, and the transmitted flux fraction is given by $F=f /\left(f_{\mathrm{qso}}^{1280} C_{q}\right)$. For a pixel at wavelength $\lambda$, the corresponding H I absorber redshift $z_{\mathrm{Ly} \alpha}$ can be inferred from $1+z_{\mathrm{Ly} \alpha}=\lambda / \lambda_{\mathrm{Ly} \alpha}$, where $\lambda_{\mathrm{Ly} \alpha}=1215.67 \AA$.

\subsection{Discussion of spectrograph resolution}

In the analysis of BOSS data [1], we encountered two main issues: determination of noise power spectrum $P^{n o i s e}(k)$ and correction of the spectrograph resolution. As the signal-to-noise ratio per pixel is much better in XQ-100 than in BOSS and since the data are at higher redshift, we can anticipate that with XQ-100 the impact of $P^{\text {noise }}(k)$ will be negligible. On the other hand, we want to study the small scales that are key for constraints on WDM. We aim at exploiting scales to $0.07 \mathrm{~s} \mathrm{~km}^{-1}$, three times smaller than with BOSS. As the spectral resolution of XSHOOTER is 3 to 5 times better 
than for BOSS, the knowledge and control of the spectrograph resolution is again a key issue of the analysis.

The spectral resolution of the UVB and VIS arms are derived from the slit widths, which were respectively $1.0^{\prime \prime}$ and $0.9^{\prime \prime}$. These slit widths provide a nominal resolving power of 4350 and 7450 as explained in [16]. However, for many observations, the seeing was smaller than the slit width, inducing an underestimate of the resolving power. To address this issue, we first determine the seeing with the raw spectra of XQ-100. The 2D spectra, as shown on Fig.2 (left plot), allow us to measure the seeing by fitting a Gaussian of the transversal distribution (i.e. orthogonal to the direction of the slit). We checked that the seeing thus measured is in good agreement with the seeing measured simultaneously at VLT (see Fig.2, right plot). On average the seeing is equal to $0.7^{\prime \prime}-0.8^{\prime \prime}$, better than the slit widths used during the observations.

As a consequence, we decided to compute our own determination of the spectral resolution, using the VLT seeing, namely (SEEING_MIN+SEEING_MAX)/2 from XQ-100_summary.fits file. We determine the resolution from the tables describing the XSHOOTER instrument (defined at [21]). When the seeing is smaller than the slit width, we substitute the slit width by the computed seeing, and compute the corresponding resolution.
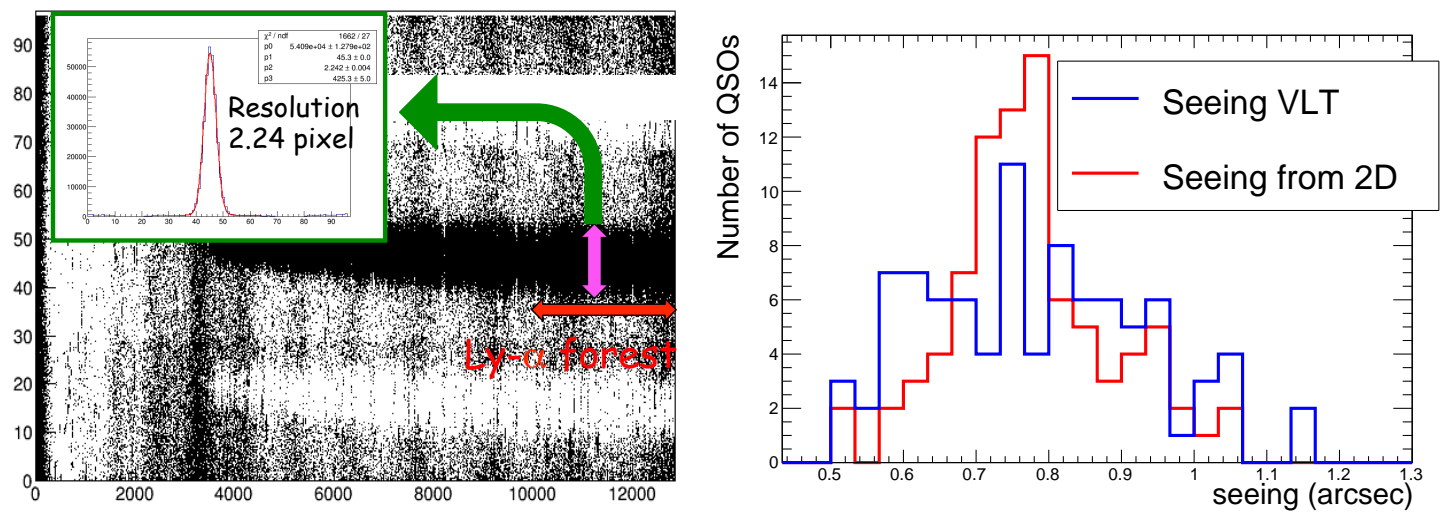

Figure 2: Left plot: example of a 2D spectrum for the UVB arm. The red arrow marks the position of the Ly- $\alpha$ forest. The transversal distribution along the purple arrow (i.e. orthogonal to the direction of the slit) allows us to visualize the PSF. The insert represents this distribution with a Gaussian fit to measure the seeing. Right plot: Distribution of the seeings for the quasars observed by XQ-100. The blue curve corresponds to the VLT measurements of the seeing during the spectroscopic observation. The red curve is derived from the $2 D$ spectra.

\subsection{Computation of the $P_{1 D}(k)$}

\subsubsection{Method}

To measure the one-dimensional power spectrum $P_{1 D}(k)$ we decompose each absorption spectrum $\delta_{\Delta v}$ into Fourier modes and estimate their variance as a function of wave number. In practice, we do this by computing the discrete Fourier transform of the flux transmission fraction $\delta=F /\langle F\rangle-1$ as described in Croft et al. [22], using a fast Fourier Transform (FFT) algorithm. In [1], we developed in parallel a likelihood approach, in a very similar way to [6]. We demonstrated that the latter method is more appropriate when noise and resolution vary from one pixel to another and when many pixels are 
masked. Because of the excellent quality of the spectrograph of XSHOOTER, a simple FFT approach can be pursued for the present analysis.

The use of a FFT requires the pixels to be equally spaced. The condition is satisfied with the SDP spectra provided by the XQ-100 pipeline [16]: the spectra are computed with a constant pixel width $\Delta[\log (\lambda)]$, and the velocity difference between pixels, i.e., the relative velocity of absorption systems at wavelengths $\lambda+\Delta \lambda / 2$ and $\lambda-\Delta \lambda / 2$, is $\Delta v=c \Delta \lambda / \lambda=c \Delta[\ln (\lambda)]$. Throughout this paper we therefore use velocity instead of observed wavelength. Similarly, the wave vector $k \equiv 2 \pi / \Delta v$ is measured in $\mathrm{s} \mathrm{km}^{-1}$.

In the absence of instrumental effects (noise and resolution of the spectrograph), the onedimensional power spectrum can be simply written as the ensemble average over quasar spectra of $P^{\text {raw }}(k) \equiv\left|\mathcal{F}\left(\delta_{\Delta v}\right)\right|^{2}$, where $\mathcal{F}\left(\delta_{\Delta v}\right)$ is the Fourier transform of the normalized flux transmission fraction $\delta_{\Delta v}$ in the quasar Ly $\alpha$ forest, binned in pixels of width $\Delta v$.

When taking into account the noise in the data, the impact of the spectral resolution of the spectrograph, the cross-correlated background due to absorption by Ly $\alpha$ and Si III and the uncorrelated background due to metal absorption such as $\mathrm{Si}$ IV or $\mathrm{C}_{\mathrm{IV}}$, the raw power spectrum is

$$
P^{r a w}(k)=\left(P^{\mathrm{Ly} \alpha}(k)+P^{\mathrm{Ly} \alpha-\mathrm{Si}_{\mathrm{II}}}(k)+P^{\text {metals }}(k)\right) \cdot W(k, R, \Delta v)+P^{\text {noise }}(k)
$$

where $W^{2}(k, R, \Delta v)$ is the window function related to spectrograph resolution.

\subsubsection{Correction of the instrumental effects}

The window function $W^{2}(k, R, \Delta v)$ corresponding to the spectral response of the spectrograph depends on the two parameters $\Delta v$ and $R$ which are respectively the pixel width and the spectrograph resolution:

$$
W(k, R, \Delta v)=\exp \left(-\frac{1}{2}(k R)^{2}\right) \times \frac{\sin (k \Delta v / 2)}{(k \Delta v / 2)} .
$$

The determination of $R$ is discussed in Sec. 2.3. We illustrate in Fig. 3 (left) the impact of the spectrograph resolution on the window function $W^{2}(k, R, \Delta v)$ for the various redshift bins considered in this work.

The noise power spectrum $P^{n o i s e}(k, z)$ is computed as a white noise, using, for each pixel, the photometric error from the SDP spectra. Fig. 3 (right) shows $P^{\text {raw }}(k)$ and $P^{n o i s e}(k)$ as a function of $k$. At small scales, i.e., high $k$ values, where $P^{\text {noise }}(k, z)$ is dominant, the raw power spectrum $P^{r a w}(k)$ asymptotically approaches our estimate of $P^{\text {noise }}(k, z)$, thus providing a clear validation of our noise model. Because of the uncertainty on the resolution correction, we limit our study to the following upper bounds in $k: 0.050,0.060$ and $0.070 \mathrm{~s} \mathrm{~km}^{-1}$ for the three redshift bins $z=3.20,3.56$ and 3.93, respectively. As a consequence, $P^{\text {noise }}(k, z)$ is always a few orders of magnitude smaller than the Ly $\alpha$ power spectrum and thus has a limited impact on the Ly $\alpha$ power spectrum measurement.

\subsubsection{Correction of the metal absorption}

The cross-correlated background due to correlated absorption by Ly $\alpha$ and $\mathrm{Si}$ III within the Ly $\alpha$ forest can be estimated directly in the power spectrum. Since Si III absorbs at $\lambda=1206.50 \AA$, just $9 \AA$ away from $\operatorname{Ly} \alpha$, it appears in the power spectrum as wiggles with a frequency corresponding to $\Delta v \sim 2271 \mathrm{~km} \mathrm{~s}^{-1}$. These oscillations are clearly seen on the BOSS power spectrum as shown on Fig.6. Their contribution cannot be isolated from the Ly $\alpha$ power spectrum and is therefore included in the model of Ly $\alpha$ power spectrum fitted to the data as done in PY15 and BP16. In a similar way, we considered a possible additional correlated absorption with Si II with a frequency of $\Delta v \sim$ 

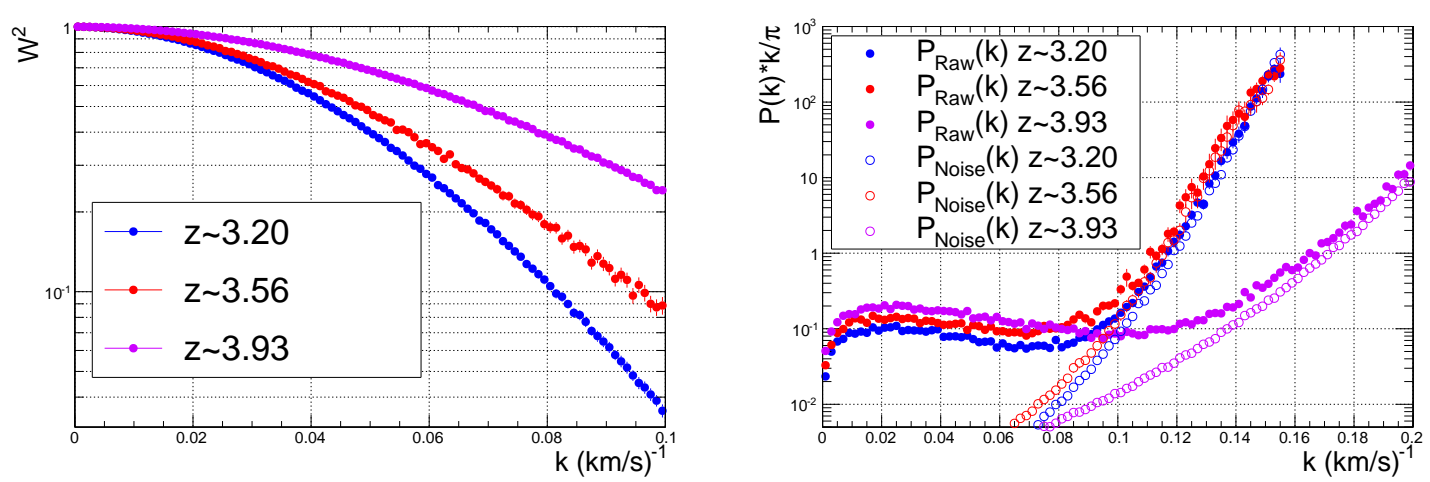

Figure 3: Left plot: Average window function $W^{2}(k, \bar{R}, \Delta v)$ for each of the three redshift bins $z=3.20$, 3.56 and 3.93 considered in this analysis. Right plot: $P^{r a w}(k)$ (large dots) and $P^{n o i s e}(k)$ (open circles) for the same redshift bins.

$5577 \mathrm{~km} \mathrm{~s}^{-1}$. In PY15 and BP16 papers, we have not significantly detected such a correlation with Si II.

The uncorrelated background due to metal absorption in the Ly $\alpha$ forest is independent of $\operatorname{Ly} \alpha$ absorption and it cannot be estimated directly from the power spectrum measured in the Ly $\alpha$ forest. In [1] we addressed this issue by estimating the background components in side bands located at longer wavelengths than the Ly $\alpha$ forest region. We apply the same technique here. We measure the power spectrum in side bands and subtract it from the Ly $\alpha$ power spectrum measured in the same gas redshift range. This method is purely statistical: for a given redshift bin, we use different quasars to compute the Ly $\alpha$ forest and the metal power spectra. It cannot be applied to the lower redshift bins of this analysis, since there are then no lower-redshift quasars from which to compute the metal contribution. Therefore, we assume that the metal power spectrum is identical for all the redshift bins, in agreement with what was shown in Fig.19 of [1].

In practice, we define one side band corresponding to the wavelength range $1270<\lambda_{\mathrm{RF}}<$ $1380 \AA$ in the quasar rest frame. The power spectrum measured in this side band includes the contribution from all metals with $\lambda_{\mathrm{RF}}>1380 \AA$, including in particular absorption from Si IV and C IV. As shown in Fig. 4 (left), the amplitude of the effect is quite comparable for three different wavelength regions, demonstrating again that we can compute a unique estimate for all redshift bins. However, we observe wiggles with a frequency corresponding to $\Delta v \sim 855 \mathrm{~km} \mathrm{~s}^{-1}$ or $\Delta \lambda \sim 20 \AA$. As this frequency varies with the tested wavelength region, and because it does not match any doublet from expected metal emission lines, its origin is likely to be instrumental. It may be a noise variation coming from the order structure of the echelle spectrum. The noise indeed increases and decreases in a periodic way due to the superposition of adjacent orders, on a scale close to $20 \AA$. As a consequence, we estimate the metal power spectrum from the wiggle-free broadband shape (blue curve on Fig. 4, right).

\subsubsection{Estimator of $P^{\mathrm{Ly} \alpha}(k)$}

We determine the 1D power spectrum, for three bins of mean redshift 3.20, 3.56 and 3.93. We compute the Fourier transform using the efficient FFTW package from [23]. The computation is done separately on each $z$-sector instead of on the entire $\operatorname{Ly} \alpha$ forest. The mean redshift of the Ly $\alpha$ absorbers in a $z$-sector determines the redshift bin to which the $z$-sector contributes. We rebin the final 

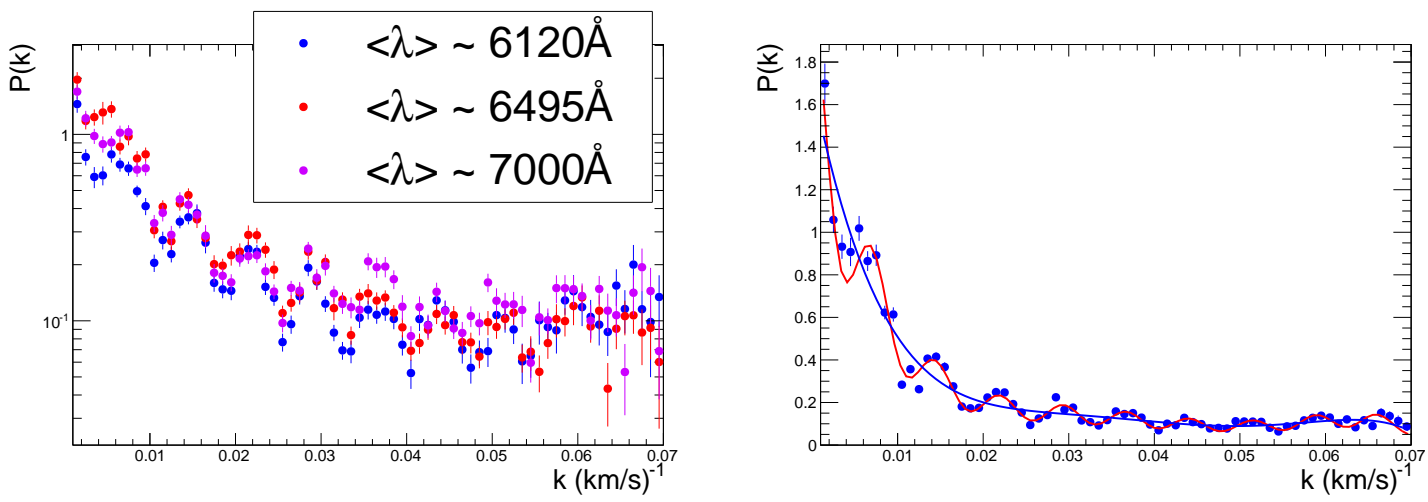

Figure 4: Measurement of the metal power spectrum $P(k)$ in the rest frame region $1270-1380 \AA$. Left plot: $P(k)$ for three wavelength bins, respectively centered on 6120, 6495 and $7000 \AA$. Right plot: instrumental-induced oscillation with a frequency $\Delta v=855 \mathrm{~km} \mathrm{~s}^{-1}$ fitted on top of the model for the metal power spectrum (red curve). The metal-only component is given by the blue curve.

power spectrum onto an evenly spaced grid in $k$-space, with $\Delta k=0.001 \mathrm{~s} \mathrm{~km}^{-1}$, giving equal weight to the different Fourier modes that enter each bin. Deriving Eq. 2.2, the final 1D power spectrum, $P_{1 D}(k)$ is obtained by averaging the corrected power spectra of all contributing $z$-sectors from all quasars, as expressed in the following estimator of $P^{\mathrm{Ly} \alpha}(k)$ :

$$
P_{1 D}(k)=\left\langle\frac{P^{r a w}(k)-P^{n o i s e}(k)}{W^{2}(k, R, \Delta v)}\right\rangle-P^{\text {metals }}(k),
$$

where \langle\rangle denotes the ensemble average over quasar spectra.

Figure 5 (left) shows the 1D Ly $\alpha$ forest power spectrum obtained with XQ-100 after subtraction of the power spectrum of the metals. It shows a good agreement with previous BOSS measurements, although the spectrographs and the data are quite different. The agreement with the other analysis of XQ-100 [2] is also remarkable, as is illustrated in Fig. 5 (right). The values $P_{1 D}(k)$, the statistical uncertainty on $P_{1 D}(k)$ and the noise power spectrum $P^{n o i s e}(k, z)$ for the three redshift bins are given in the appendix in Tab. 5. In this study, we have assumed that the estimated errors are uncorrelated.

\section{Combining data}

In this section, we first briefly introduce the data sets that we use to constrain the sum of neutrino masses and the mass of WDM. We then present the hydrodynamical simulations we ran to interpret the 1D Ly $\alpha$ forest power spectrum, and we explain the methodology followed in this paper, which builds upon the one we developed for PY15 and BP16.

\subsection{Data}

\subsubsection{Ly $\alpha$}

As our large-scale structure probe, we use the 1D Ly $\alpha$-flux power spectrum measurement from the first release of BOSS quasar data [1]. The data consist of a sample of 13821 spectra selected from the larger sample of about 60000 quasar spectra of the SDSS-III/BOSS DR9 [24-29] on the basis of their high quality, high signal-to-noise ratio $(>2)$ and good spectral resolution $\left(<85 \mathrm{~km} \mathrm{~s}^{-1}\right.$ on 

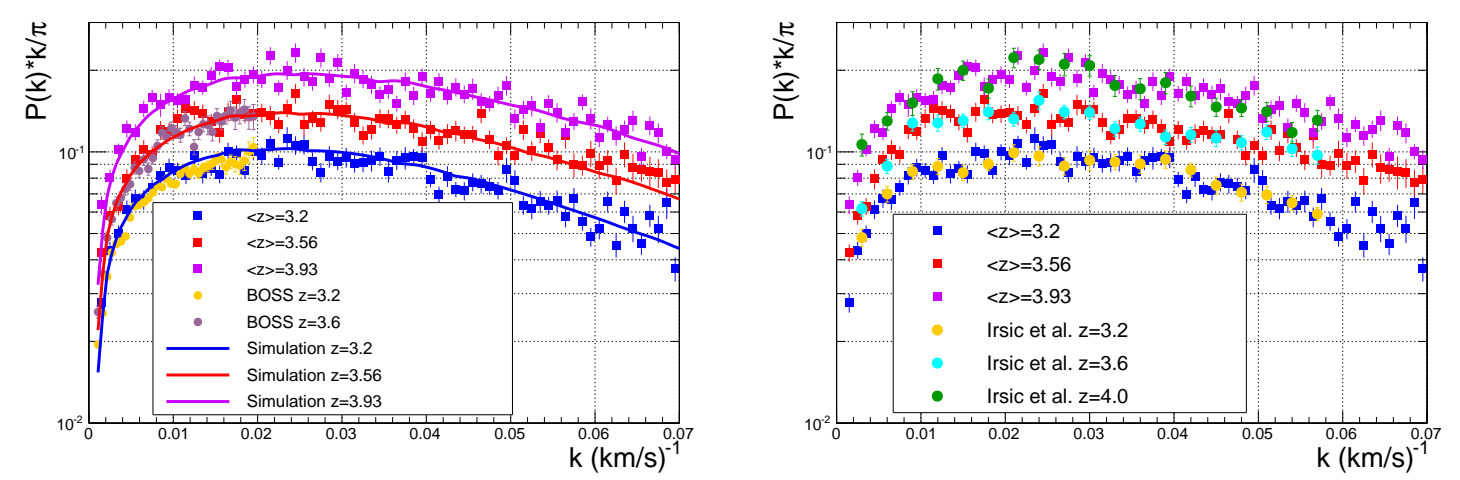

Figure 5: Left plot: $1 D$ Lya forest power spectrum from XQ-100 spectra computed as explained in this paper and compared to BOSS power spectrum published in [1]. The curves show the power spectrum derived from the simulations of [17], corresponding to the "best guess" configuration $\left(\sigma_{8}=\right.$ $0.83, n_{s}=0.96$, and $\Omega_{m}=0.31$ ). The values of the power spectra are available in the appendix in Tab. 5. Right plot: Same 1D Ly $\alpha$ forest power spectrum from XQ-100 data, here compared to the power spectrum computed in [2] for the same data and three similar redshift bins.

average over a quasar forest). We do the analysis on 420 Ly $\alpha$ data points, consisting of 12 redshift bins over $2.1<z<4.5$ and $35 k$ bins with $k \leq 0.020 \mathrm{~km} \mathrm{~s}^{-1}$ as shown on Fig. 6 .

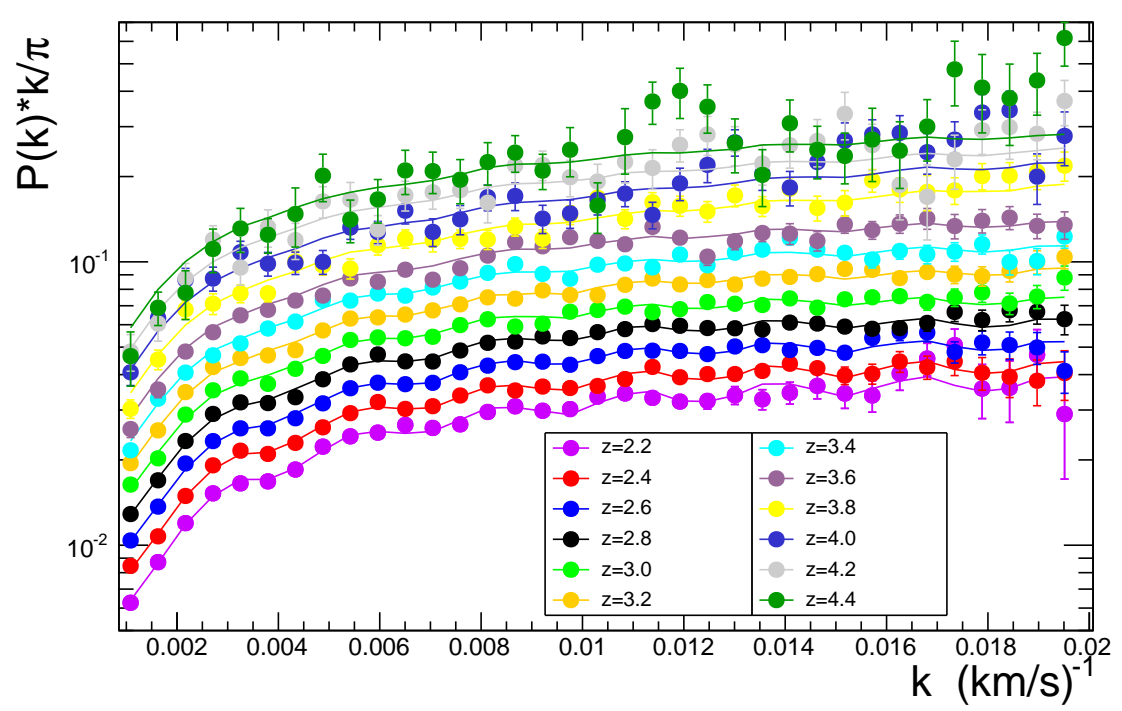

Figure 6: $1 D$ Lya forest power spectrum from the SDSS-III/BOSS DR9 data. The solid curves show the best-fit model when considering Ly $\alpha$ data alone. The oscillations arise from Ly $\alpha$-Si III correlations, which occur at a wavelength separation $\Delta \lambda=9.2 \AA$.

We complement the BOSS 1D Ly $\alpha$-flux power spectrum with the XQ-100 [16] power spectrum. First, we use the power spectrum computed in Sec. 2, consisting of three redshift bins at $z=3.2,3.56$ and 3.93, and, respectively, 50, 60 and $70 k$ bins corresponding to $k \leq 0.050,0.060$ and $0.070 \mathrm{~s} \mathrm{~km}^{-1}$ 
as shown in Fig. 7 (left). Then, for the study of WDM in Sec 5, we also include in our likelihood the XQ-100 power spectrum measured by [2]. This data set, shown in Fig. 7 (right), consists of 7 redshift bins with $2.9<z<4.3$ and $19 k$ bins with $k \leq 0.057 \mathrm{~s} \mathrm{~km}^{-1}$.

Finally, to the BOSS+XQ-100 data set, we further add the power spectrum measured by [4] with the high-resolution HIRES/MIKE spectrographs. We use the lowest two redshift bins, $z=4.2$ and $z=4$.6. The power spectrum shown in Fig. 7 (left) was obtained by combining in quadrature the HIRES and MIKE for $9 k$ bins with $k \leq 0.080 \mathrm{~s} \mathrm{~km}^{-1}$. The highest-redshift snapshot that we extracted from our simulations (see Sec. 3.2) is at $z=4.6$, which prevents us from using the other two redshift bins $(z=5.0$ and $z=5.4)$ of the HIRES/MIKE data.
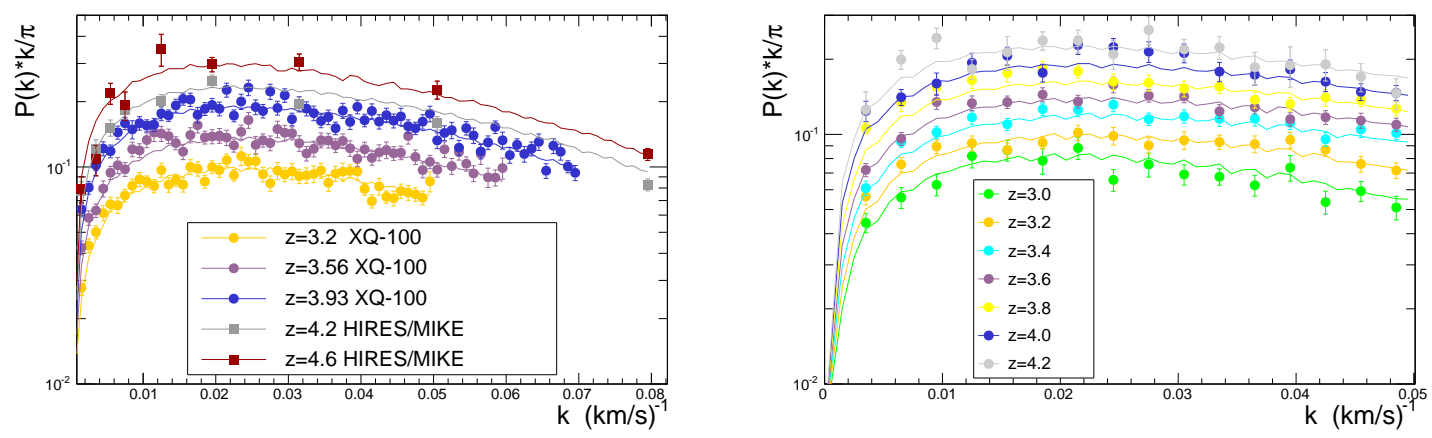

Figure 7: $1 D$ Ly $\alpha$ forest power spectrum from XQ-100 data and HIRES/MIKE data. The solid curves show the best-fit model when considering Lya data from BOSS and XQ-100. The oscillations arise from Ly $\alpha$-Si III correlations, which occur at a wavelength separation $\Delta \lambda=9.2 \AA$ and are driven by the BOSS data. The left plot corresponds to the analysis of the power spectrum presented in this paper. The right plot is the power spectrum measured in [2].

\subsubsection{Cosmic microwave background}

As in PY15, we use cosmic microwave background (CMB) data and results from the full Planck mission [12]. We consider several subsets of Planck data. The base configuration, denoted 'TT+lowP' as in [12], uses the TT spectra at low and high multipoles and the polarization information up to multipoles $\ell=29$ ('lowP'). We also use at times the configuration based on TT, TE and EE spectra, along with the low-multipole polarization, denoted 'TT+TE+EE+lowP'.

\subsubsection{Baryon acoustic oscillations}

We occasionally combine CMB data with measurements of the BAO scale by 6dFGS [30], SDSS main galaxy sample [31], BOSS-LOWZ [32] and CMASS-DR11 [32]. Theses measurements are henceforth globally denoted 'BAO'. The additional constraints that these measurement provide on cosmological parameters are included in the present work with their full correlation with CMB data. Both CMB and BAO constraints are taken from the Markov Chains publicly available through the official Planck Legacy Archive at http://pla.esac.esa.int.

\subsection{Simulations}

To predict the Ly $\alpha$ flux power spectrum, we use the set of simulations extensively described in PY15 and BP16. The simulations are run using a parallel tree smoothed particle hydrodynamics (treeSPH) code GADGET-3, an updated version of the public code GADGET-2 [33, 34]. The simulations are 
started at $z=30$, with initial transfer functions and power spectra computed with CAMB [35], and initial particle displacements generated with second-order Lagrangian Perturbation Theory 2LPT ${ }^{1}$. We include three particle types: collisionless dark matter, gas, and, when relevant, degenerate-mass neutrinos. We showed in [19] that considering inverted or normal neutrino mass hierarchy yields a flux power spectrum that differs by less than $0.05 \%$ from the degenerate-mass scenario, a level ten times lower than the statistical uncertainties in the simulation and almost two orders of magnitude below the data uncertainties. The degenerate-mass hypothesis is thus highly justified. The simulations cover the volume of a periodic $100 \mathrm{Mpc} / h$ box containing the equivalent of $3072^{3}$ particles of each type. Following a method originally suggested in [36], we obtain this resolution by splicing together large-volume and high-resolution simulations, using a transition simulation that corrects the large box for its lack of coupling between small and large modes, and the high-resolution simulation for its small volume. We studied the accuracy of the splicing technique in [19] and we leave parameters free when fitting the data to account for residual biases.

The cosmological parameters are centered on the Planck 2013 best-fit values [37]. Using simulations where one or two parameters at a time are given off-centered values, we compute first and second-order derivatives of the Ly $\alpha$ flux power spectrum with respect to each parameter, which we use to derive a second-order Taylor expansion of the predicted Ly $\alpha$ flux power spectrum. The cosmological parameters cover the range $H_{0}=67.5 \pm 5 \mathrm{~km} \mathrm{~s}^{-1} \mathrm{Mpc}^{-1}, \Omega_{M}=0.31 \pm 0.05, n_{s}=0.96 \pm 0.05$, $\sigma_{8}=0.83 \pm 0.05$. In all the runs, we keep $\Omega_{b}=0.0221$. While our central simulation assumes massless neutrinos, some runs include neutrinos with masses $\sum m_{v}=0.4$ or $0.8 \mathrm{eV}$. Where WDM is assumed, the dark matter particles are thermal relics with masses $m_{X}=2.5$ or $5.0 \mathrm{keV}$. All our simulation runs start at $z=30$, with initial conditions having the same random seed. Snapshots are produced at regular intervals in redshift from $z=4.6$ to 2.2, with $\Delta z=0.2$.

We test the influence of assumptions on the IGM astrophysics by running simulations for central and offset values of relevant parameters. The photo-ionization rate of each simulation is fixed by requiring the effective optical depth at each redshift to follow the empirical law $\tau_{\text {eff }}(z)=A^{\tau}(1+z)^{\eta^{\tau}}$, with $A^{\tau}=0.0025 \pm 0.0020$ and $\eta^{\tau}=3.7 \pm 0.4$ in agreement with [38]. This renormalization is done at the post-processing stage, as justified in [39], allowing us to test the impact of different scalings without running new simulations. The IGM temperature-density relation $T=T_{0} \Delta^{\gamma-1}$ is obtained using simulations ran for $\gamma(z=3)=1.3 \pm 0.3$ and $T_{0}(z=3)=14000 \pm 7000 \mathrm{~K}$. We use the quickLy $\alpha$ option to convert gas particles with overdensities exceeding $10^{3}$ and temperature below $10^{5} \mathrm{~K}$ into stars.

\subsection{Methodology}

The analysis of the data is done with a frequentist approach. We showed in [40] that the constraints obtained with either a frequentist or a Bayesian (MCMC) approach were in excellent agreement. The large number of nuisance parameters that are now included in the fit, however, prevents the use of a Bayesian method where convergence is then hard to reach.

The parameters that are floated in the minimization procedure belong to three categories. The first category models a flat $\Lambda \mathrm{CDM}$ cosmology with free $H_{0}, \Omega_{M}, n_{s}, \sigma_{8}$ and either $\sum m_{v}, m_{X}$ or $m_{s}$. The second category describes the IGM, letting free the parameters described in table 1 , namely $T_{0}$, $\gamma, \eta^{T_{0}}(z<3), \eta^{T_{0}}(z>3), \eta^{\gamma}, A^{\tau}, \eta^{\tau}$ and two amplitudes for the correlated absorption of Ly $\alpha$ with $\mathrm{Si}$ III or Si II. In particular, to take into account the redshift evolution of $T_{0}(z), \gamma(z)$, we modeled them using a single power law for $\gamma$ and a broken power law at $z=3$ for $T_{0}$ as explained in [19].

Finally, the last category groups all nuisance parameters that allow us to account for uncertainties or corrections related to noise in the data, spectrograph resolution, modeling of the IGM, residual

\footnotetext{
${ }^{1}$ http://cosmo.nyu.edu/roman/2LPT/
} 
bias in the slicing technique, supernova and AGN feedbacks, and redshift of reionization. Details on the fit parameters and on the dependance with scale and redshift of the nuisance parameters can be found in [19].

\section{Constraints on $\Lambda \mathrm{CDM} v$}

In this section, we present the cosmological results of our analysis considering $\Lambda \mathrm{CDM} v$ cosmology with standard active neutrinos. The analysis extends the method presented in PY15 to the XQ-100 data. We first describe the constraints obtained with Ly $\alpha$ alone and we then add CMB and BAO data.

\section{1 $\Lambda \mathrm{CDM} v \operatorname{cosmology}$ from $\operatorname{Ly} \alpha$ data alone}

The results for Ly $\alpha$ alone are shown in columns (1) to (3) of Tab. 3. Column (1) recalls the results of PY15 for BOSS Ly $\alpha$ alone. The maximization of the likelihood with Ly $\alpha$ data, imposing a Gaussian constraint $H_{0}=67.3 \pm 1.0$, gives a best-fit value of the sum of the neutrino masses $\sum m_{v}$ equal to $0.41 \mathrm{eV}$ and compatible with 0 at about $1 \sigma$. The upper bound on $\sum m_{v}$ is thus $1.1 \mathrm{eV}$ (95\% C.L.).

In column (2) of Tab. 3, we present the results with XQ-100 alone, using $P(k)$ measured with the method described in Sec. 2 . The upper bound on $\sum m_{v}$ is $1.2 \mathrm{eV}$ (95\% C.L.), compatible with the upper bound from BOSS alone.

The other astrophysical and cosmological parameters are in very good agreement between BOSS-alone and XQ-100-alone. The largest differences observed are for $T_{0}$ and $\sigma_{8}$. They represent respectively $1.7 \sigma$ and $1.4 \sigma$. However, the comparison of BOSS and XQ-100 and the interpretation of differences in the best-fit parameters may be delicate as all the variables are correlated. In particular, we illustrate in Fig. 8 (left) the strong anti-correlation between $T_{0}$ and $\gamma$. As a result, a low value of $\gamma$ pushes $T_{0}$ to high values. In this neutrino-mass-oriented analysis, the likelihood is built in such a way as not to be too sensitive to underlying assumptions on IGM parameters, which we here treat as nuisance parameters. The shapes of $T_{0}(z)$ and $\gamma(z)$ are let free in the maximization of the likelihood as explained in Sec. 3.3.

Finally, by combining BOSS and XQ-100, see column (3), the best-fit value of $\sum m_{v}$ decreases to $0.34 \mathrm{eV}$ and the upper bound on $\sum m_{v}$ tightens to $0.8 \mathrm{eV}$ (95\% C.L.). The fitted values of astrophysical and nuisance parameters are all well within the expected range. The neutrino mass is correlated to $\sigma_{8}(-26 \%), n_{s}(19 \%)$ and $\Omega_{m}(33 \%)$. Correlations between all other cosmological parameters have smaller amplitudes.

\section{2 $\Lambda \mathrm{CDM} v \operatorname{cosmology}$ from $\operatorname{Ly} \alpha$ data and other probes}

We now combine the Ly $\alpha$ likelihood (imposing no constraint on $H_{0}$ ) with the likelihood of Planck 2015 data that we derive from the central values and covariance matrices available in the official 2015 Planck repository. As in the previous section, we focus on the base $\Lambda \mathrm{CDM} v \operatorname{cosmology}$. Column (4) of Tab. 3 shows the results for the combined set of Ly $\alpha$ (BOSS and XQ-100) and the base configuration we chose for Planck data, i.e. (TT+lowP) (cf. details in Sec. 3). In column (5), we extend the $\mathrm{CMB}$ measurements to (TT+TE+EE+lowP) and we add BAO data.

We illustrate in Fig. 8 (right) the main 2D contours on cosmological parameters. The small tension on $n_{s}$ between Ly $\alpha$ and Planck data, observed in [19,40], is still present when including XQ-100 data, although its significance is reduced to $1.7 \sigma$. We demonstrated in PY15, anyhow, that the tension on $n_{s}$ has little effect on the constraint on $\sum m_{v}$ because of the mild correlation between these two parameters (19\% in Ly $\alpha,-45 \%$ in Planck TT+lowP). As was already seen in PY15, $\sum m_{v}$ is mostly correlated to $\sigma_{8}\left(-26 \%\right.$ in $\mathrm{Ly} \alpha,-95 \%$ in Planck TT+lowP) and to $\Omega_{m}(19 \%$ in $\mathrm{Ly} \alpha, 92 \%$ in Planck TT+lowP). 
Table 3: Best-fit value and 68\% confidence levels of the cosmological parameters of the model fitted

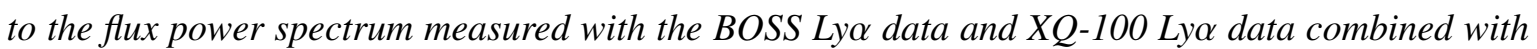
several other data sets. Column (1) shows the results of PY15 with BOSS alone [19]. Column (2) shows the results with XQ-100 alone, using $P(k)$ measured with the method described in Sec. 2 . Column (3) shows the results for the combined fit of BOSS and XQ-100. For columns (1-3), we used a Gaussian constraint, $H_{0}=67.3 \pm 1.0$. The following columns are obtained by combining Ly $\alpha$ with $C M B$ and $B A O$.

\begin{tabular}{|c|c|c|c|c|c|}
\hline Parameter & $\begin{array}{c}\text { Ly } \alpha \\
\text { BOSS } \\
+H_{0}^{\text {Gaussian }}\end{array}$ & $\begin{array}{c}\text { Ly } \alpha \\
\text { XQ-100 } \\
+H_{0}^{\text {Gaussian }}\end{array}$ & $\begin{array}{c}\text { Ly } \alpha \\
\text { BOSS }+ \text { XQ-100 } \\
+H_{0}^{\text {Gaussian }}\end{array}$ & $\begin{array}{c}\text { Ly } \alpha \\
\text { BOSS + XQ-100 } \\
+ \text { Planck } \\
(\mathrm{TT}+\text { lowP }) \\
\\
\text { (4) }\end{array}$ & $\begin{array}{c}\text { Ly } \alpha \\
\text { BOSS + XQ-100 } \\
+ \text { Planck } \\
(\mathrm{TT}+\mathrm{TE}+\mathrm{EE}+\text { lowP }) \\
+ \text { BAO } \\
(5)\end{array}$ \\
\hline$T_{0}(\mathrm{z}=3)\left(10^{3} \mathrm{~K}\right)$ & $8.9 \pm 3.9$ & $21.4 \pm 6.0$ & $14.7 \pm 3.3$ & $16.1 \pm 2.5$ & $16.5 \pm 2.5$ \\
\hline$\gamma$ & $0.9 \pm 0.2$ & $0.88 \pm 0.5$ & $1.0 \pm 0.2$ & $0.7 \pm 0.2$ & $0.7 \pm 0.2$ \\
\hline$\sigma_{8}$ & $0.831 \pm 0.031$ & $0.738 \pm 0.059$ & $0.783 \pm 0.023$ & $0.837 \pm 0.017$ & $0.830 \pm 0.015$ \\
\hline$n_{s}$ & $0.939 \pm 0.010$ & $0.920 \pm 0.023$ & $0.950 \pm 0.008$ & $0.962 \pm 0.004$ & $0.961 \pm 0.004$ \\
\hline$\Omega_{m}$ & $0.293 \pm 0.014$ & $0.317 \pm 0.024$ & $0.282 \pm 0.012$ & $0.288 \pm 0.013$ & $0.310 \pm 0.007$ \\
\hline$H_{0}\left(\mathrm{~km} \mathrm{~s}^{-1} \mathrm{Mpc}^{-1}\right)$ & $67.3 \pm 1.0$ & $67.2 \pm 1.0$ & $67.1 \pm 1.0$ & $69.1 \pm 1.0$ & $67.7 \pm 0.6$ \\
\hline$\sum m_{v}(\mathrm{eV})$ & $<1.1(95 \% \mathrm{CL})$ & $<1.2(95 \% \mathrm{CL})$ & $<0.8(95 \% \mathrm{CL})$ & $<0.14(95 \% \mathrm{CL})$ & $<0.14(95 \% \mathrm{CL})$ \\
\hline
\end{tabular}
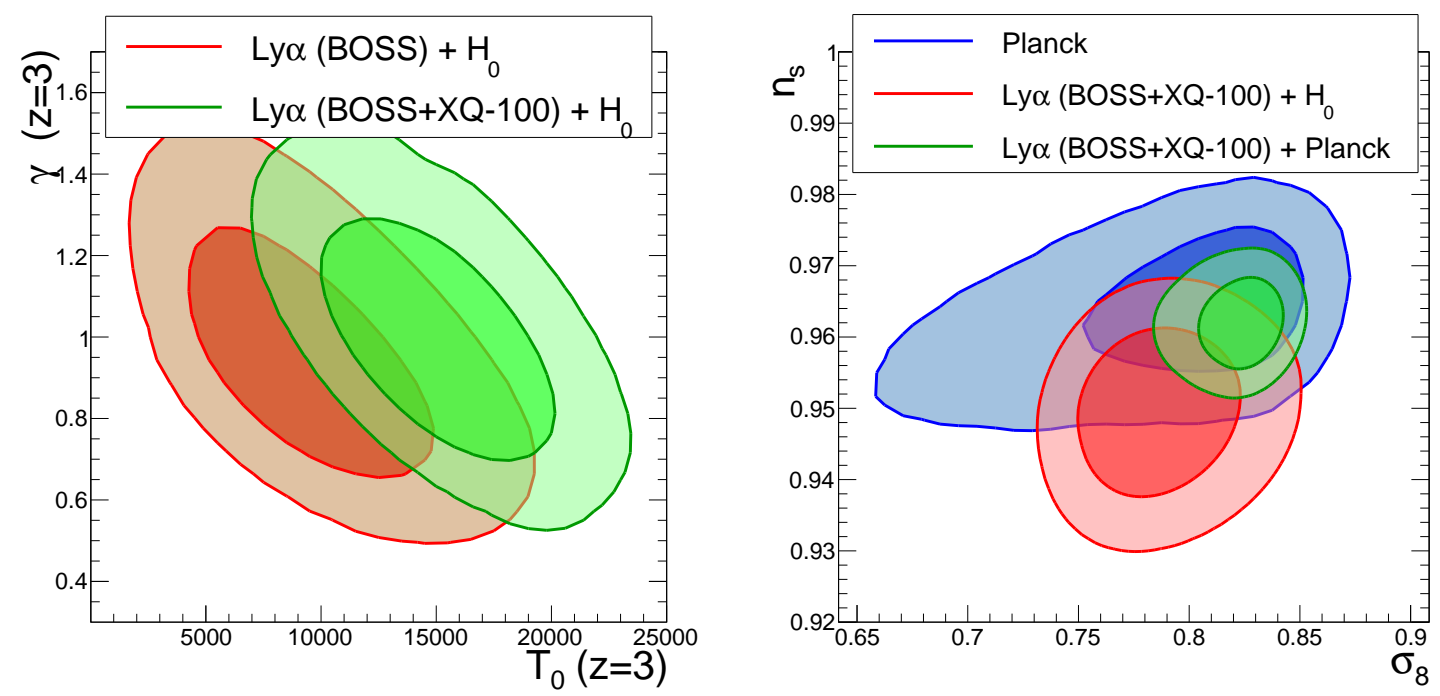

Figure 8: $2 D$ confidence level contours for the $\left(T_{0}, \gamma\right)$ astrophysical parameters defined at redshift $z=3$ and for the $\left(\sigma_{8}, n_{s}\right)$ cosmological parameters. Left Plot: $68 \%$ and $95 \%$ confidence contours obtained for the BOSS Ly $\alpha$ data with a Gaussian constraint $H_{0}=67.3 \pm 1.0 \mathrm{~km} \mathrm{~s}^{-1} \mathrm{Mpc}^{-1}$ (red), and

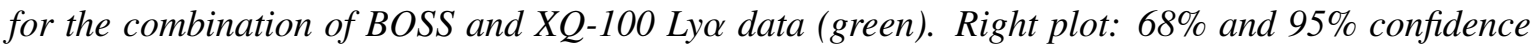
contours obtained for the BOSS and XQ-100 Ly data with a Gaussian constraint $H_{0}=67.3 \pm$ $1.0 \mathrm{~km} \mathrm{~s}^{-1} \mathrm{Mpc}^{-1}$ (red), for the Planck 2015 TT+lowP data (blue) and for the combination of Lya and Planck 2015 (green). 
Fig. 9 shows that the combination of Planck and Ly $\alpha$ is a very efficient way of constraining cosmological parameters, especially $\sum m_{v}$. As we explained in PY15, Ly $\alpha$ data constrain $\Omega_{m}$ and $\sigma_{8}$ largely independently of $\sum m_{v}$ because they have different impacts on the shape of the power spectrum. On the other hand, in CMB data, $\sum m_{v}$ is strongly correlated with $\Omega_{m}$ and $\sigma_{8}$. For the Planck constraints, high $\sum m_{v}$ corresponds to low $\sigma_{8}$ because of the suppression of power on small scales by neutrino free streaming. The positive correlation between $\Omega_{m}$ and $\sum m_{v}$ is more subtle: with $\Omega_{c} h^{2}$ and $\Omega_{b} h^{2}$ well constrained by the acoustic peaks, raising $\sum m_{v}$ increases the matter density at low redshift after neutrinos become non-relativistic, and within $\Lambda C D M$ this requires a decrease in $h$ to maintain the well determined angular diameter distance to last scattering, and this in turn corresponds to higher $\Omega_{m}$ (see, e.g., $§ 6.4$ of [12]). The end result is that the Ly $\alpha$ and Planck contours intersect only near $\sum m_{v}=0$. By combining (BOSS and XQ-100) Ly $\alpha$ and Planck TT+lowP data, we constrain $\sum m_{v}$ to be less than $0.14 \mathrm{eV}$ at $95 \%$ C.L.. Finally, adding polarization or BAO to the Planck + Ly $\alpha$ contours does not lead to significant further improvement of the constraints as shown in column (5) of Tab 3.
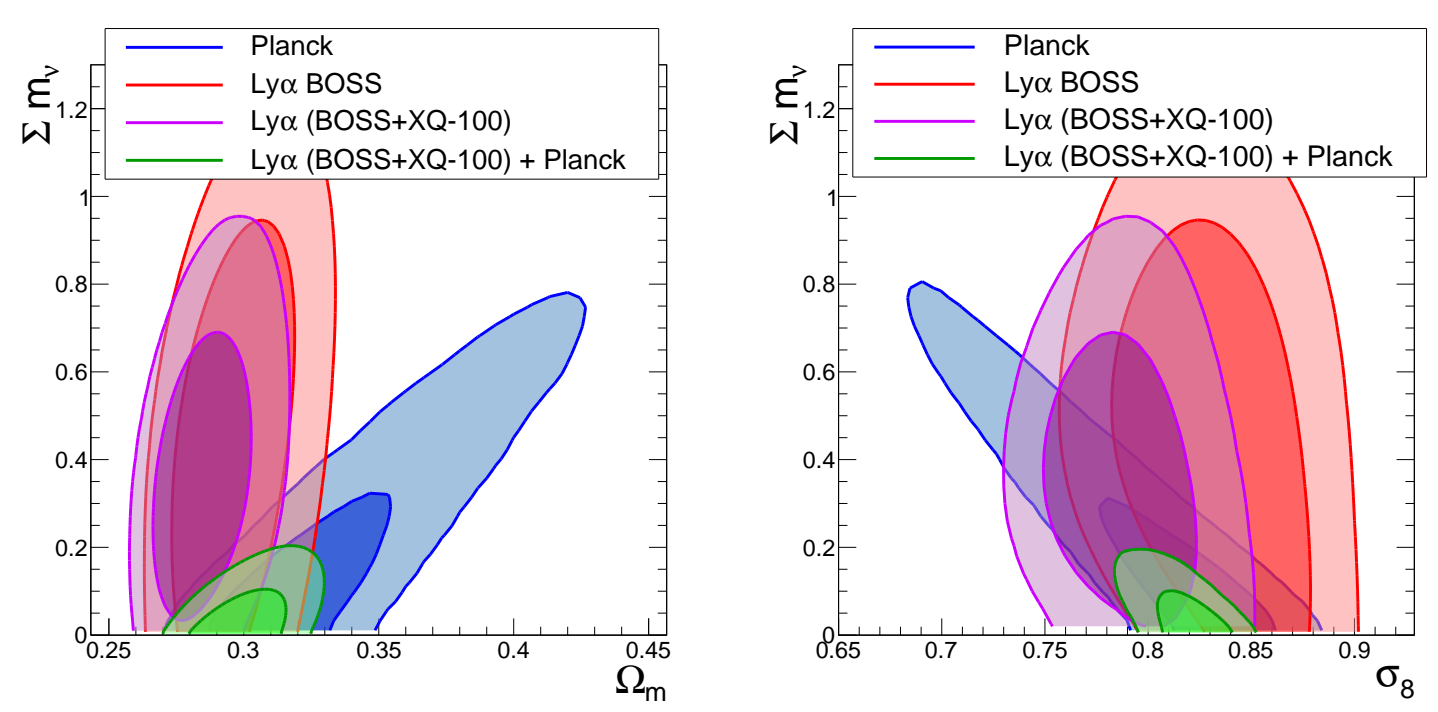

Figure 9: The 68\% and 95\% 2D confidence level contours for Planck $2015 T T+$ lowP data (blue), BOSS data with a Gaussian constraint on $H_{0}$ (red), then by adding XQ-100 data to BOSS data (magenta) and finally by combining BOSS, XQ-100 and Planck (green). Left plot: $2 D$ confidence level contours for $\left(\Omega_{m}, \sum m_{v}\right)$. Right plot: $2 D$ confidence level contours for $\left(\sigma_{8}, \sum m_{v}\right)$.

The current limit on $\sum m_{v}$ is a bit looser that the $0.12 \mathrm{eV}$ bound of PY15, whereas we note a small improvement in the Ly- $\alpha$ alone constraint with the inclusion of XQ-100 (cf. Sec. 4.1). The origin of this result can be understood when plotting the $\chi^{2}$ profile for three combinations of data sets (cf. Fig. 10 (right)). The minimum for the BOSS Ly $\alpha+$ Planck configuration (red curve) occurs for $\sum m_{v}<0$. The fact that the CMB data sets have their minimum in the unphysical (negative $\sum m_{v}$ ) region was already discussed in [41]. In the results presented in Tab. 3, the limit on the total neutrino mass is derived by computing the probability of $\Delta \chi^{2}\left(\sum m_{v}\right)=\chi^{2}\left(\sum m_{v}\right)-\chi^{2}\left(\sum m_{v}=0\right)$ with one degree of freedom. In the case of the (BOSS+XQ-100) Ly $\alpha+$ Planck configuration, the curvature of the $\chi^{2}$ profiles stays almost unchanged, but the position of the minimum is shifted closer to the physical $\left(\sum m_{v}>0\right)$ region, causing a looser bound on $\sum m_{v}$.

The shift of the $\chi^{2}$ minimum into the physical region can be easily explained. By adding XQ- 
100 , the combined value of $\sigma_{8}$ is smaller compared to BOSS alone (see first and second columns of Tab 3). The two parameters $\sigma_{8}$ and $\sum m_{v}$ are anti-correlated for Planck data as shown on Fig. 10 (left). As a consequence, XQ-100 pushes toward higher $\sum m_{v}$.

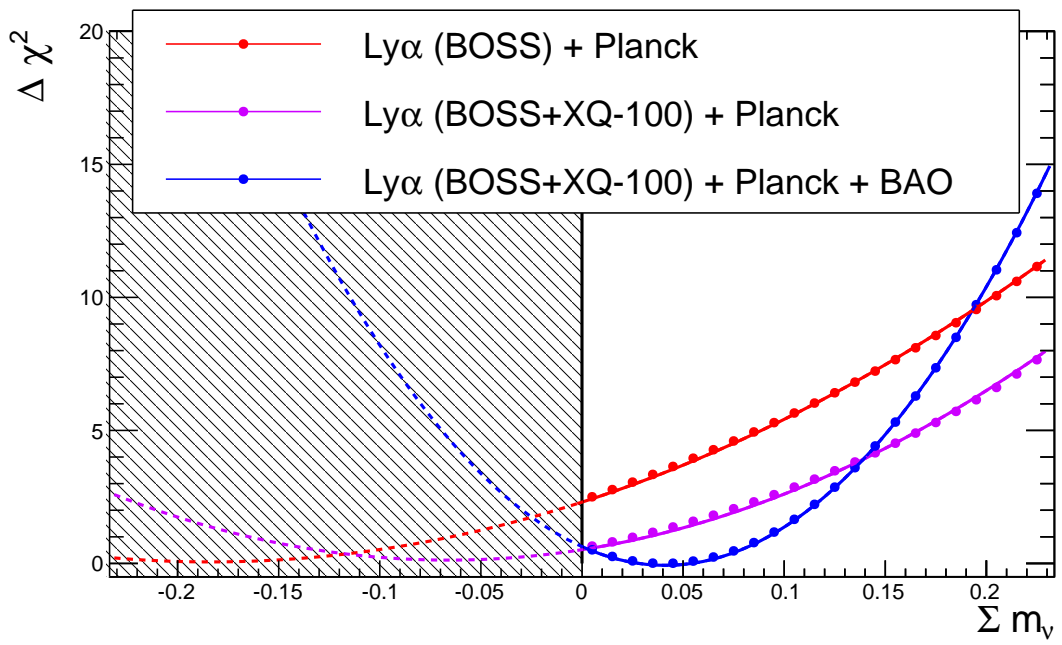

Figure 10: $\Delta \chi^{2}$ profile as a function of $\sum m_{v}$ for the three configurations $(L y \alpha(B O S S)+$ Planck), $($ Ly $\alpha(B O S S+X Q-100)+$ Planck $)$ and $($ Ly $\alpha(B O S S+X Q-100)+$ Planck + BAO $)$. Each point is the $\Delta \chi^{2}$ obtained after a maximization of the total likelihood over the other free parameters. The points are fitted by a parabola and extrapolated into the negative region as proposed in [41].

Finally, as shown in Fig. 10 (right) (blue curve), adding BAO data definitively forces the minimum back into the physical $\left(\sum m_{v}>0\right)$ region. Hence, despite a much more constraining set of data (the limit at $3 \sigma$ or more is the tightest when all $\operatorname{Ly} \alpha, \mathrm{CMB}$ and $\mathrm{BAO}$ data are included), we obtain a limit on the total neutrino mass of $\sum m_{v}<0.14 \mathrm{eV}$ at $95 \% \mathrm{CL}$, identical to the limit with $\operatorname{Ly} \alpha$ and Planck.

\section{Constraints on $\Lambda \mathrm{WDM}$}

In this section, we present the cosmological results of our analysis on $\Lambda \mathrm{WDM}$ cosmology with thermal relics and non-resonantly produced neutrinos. We first extend the method explained in BP16 to XQ-100 data. We then compare the constraints obtained with the Ly $\alpha$ power spectrum measured in this study to those obtained with the power spectrum of [2].

\section{1 $\Lambda$ WDM cosmology with XQ-100}

To probe $\Lambda \mathrm{WDM}$ cosmology, we follow a similar approach as described in Sec.3.3. The only significant difference is related to the uncertainty on the reionization history of the universe. As the redshift at which the UV background onsets affects the Jeans smoothing scale of the baryon gas [42] in a manner similar to the free streaming scale of warm dark matter particles, altering the reionization redshift $z_{\star}$ impacts the definition of the WDM free streaming scale. Fig. 13 of [43] shows that an increase in the redshift of reionization from $z_{\star}=7$ to 17 suppresses the Ly $\alpha$ flux power spectrum in the largest $k$-modes present in the BOSS data $\left(k \sim 0.02 \mathrm{~s} \mathrm{~km}^{-1}\right)$ by about $1 \%$ at $z=2.1$ and $4 \%$ at $z=4.0$. For XQ-100 where the power spectrum is measured at much smaller scales, $\left(k \sim 0.07 \mathrm{~s} \mathrm{~km}^{-1}\right.$ at $\left.z=4.0\right)$, 
we are very sensitive to such an effect. Using the study of reionization by [43], we model the effect of reionization over the power spectrum and we introduce a nuisance parameter representing $z_{\star}$. This new parameter is let free in the likelihood with a constraint $z_{\star}=9.0 \pm 1.5$. The central value and range of this external constraint are defined in order to encompass the most recent measurements of the redshift of reionization $[12,44,45]$.

A fit to the power spectrum of XQ-100 Ly $\alpha$ forest, assuming the expansion rate value $H_{0}=$ $67.3 \pm 1.0 \mathrm{~km} \mathrm{~s}^{-1} \mathrm{Mpc}^{-1}$ issued by [12], yields a lower bound $m_{X}>2.08 \mathrm{keV}$ (95\% C.L.) for thermal relics and $m_{s}>10.2 \mathrm{keV}$ for Dodelson-Widrow [46] sterile neutrinos (95\% CL). These bounds are roughly twice lower than the bounds given with BOSS alone by BP15 in Fig. 11 (left) and Tab. 4.

When combining XQ-100 with BOSS Ly $\alpha$ forest power spectra, the 95\% C.L. limit is slightly improved compared to the one from BOSS alone. It increases from $m_{X} \gtrsim 4.09 \mathrm{keV}$ to $m_{X} \gtrsim 4.17 \mathrm{keV}$. The $3 \sigma$ bound shows a more significantly improvement, increasing from $m_{X} \gtrsim 2.74 \mathrm{keV}$ for BOSS alone to $m_{X} \gtrsim 3.10 \mathrm{keV}$ for the combined BOSS+XQ-100 data set.
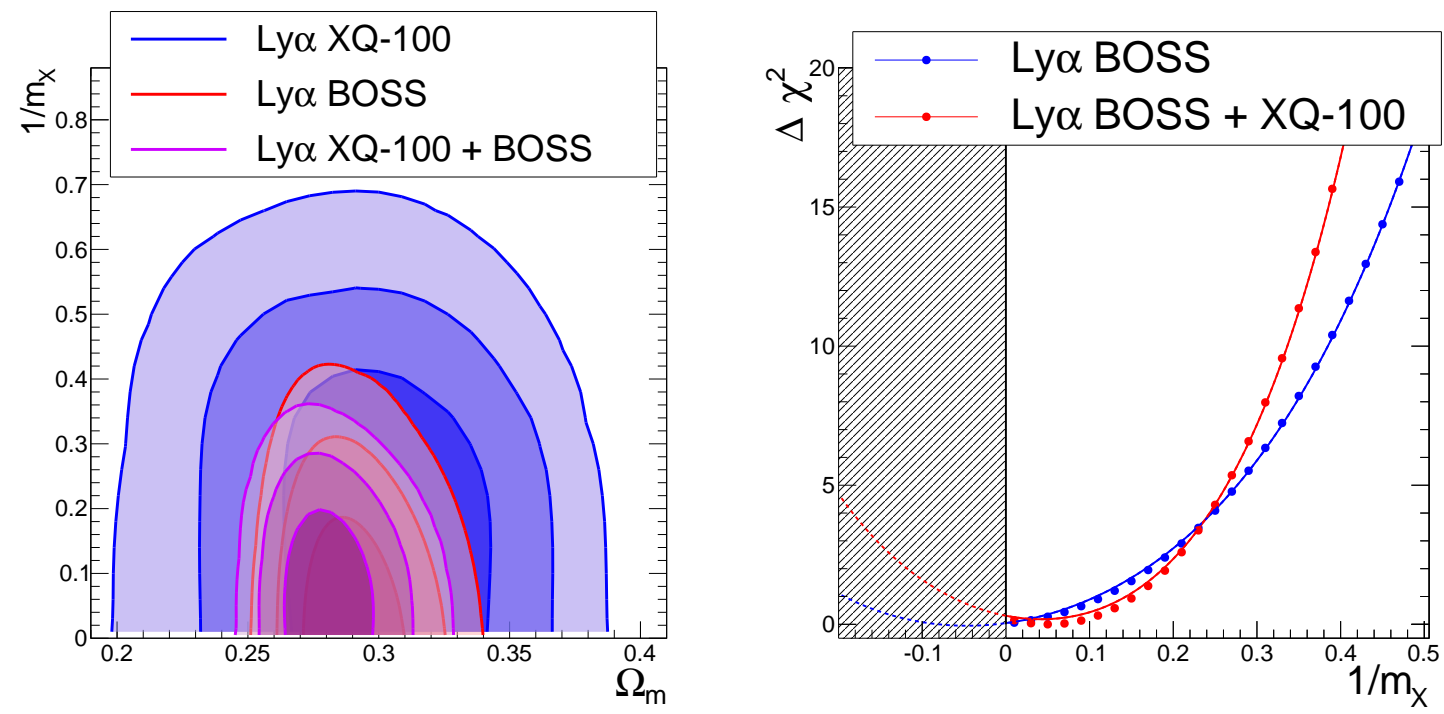

Figure 11: Left plot : $2 D$ confidence level contours for the $\left(\Omega_{m}, 1 / m_{x}\right)$ cosmological parameters. The

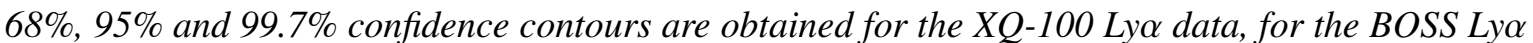
data separately and for the combination of XQ-100 and BOSS. Right plot: $\Delta \chi^{2}$ profile as a function of $1 / m_{x}$ for the two configurations: BOSS alone and the combination of BOSS and XQ-100. Each point is the $\Delta \chi^{2}$ obtained after a maximization of the total likelihood over the other free parameters.

The reason of this improvement is clearly illustrated in Fig. 11 (right), which shows the $\chi^{2}$ profile for two combinations of data sets: BOSS alone and BOSS+XQ-100. Clearly, the curvature increases by adding XQ-100 but the position of the minimum is shifted into the physical $\left(1 / m_{x}>0\right)$ region, explaining a small improvement of the $95 \%$ C.L bound and a larger improvement at higher significance $\left(3 \sigma\right.$ or more). Fitting the $\chi^{2}$ profile by $\Delta \chi^{2}\left(1 / m_{X}\right)=\chi_{0}^{2}+\left(1 / m_{x}-1 / m_{x_{0}}\right)^{2} / \sigma^{2}+$ $\alpha \cdot\left(1 / m_{x}-1 / m_{x_{0}}\right)^{4}$, the parameter $\sigma$ provides an estimator of the statistical sensitivity on $1 / m_{X}$. The combination with XQ-100 allows us to reduce $\sigma$ from 0.15 to 0.12 , representing a $25 \%$ gain in statistical sensitivity.

Although we believe that a Gaussian constraint with a sigma of 1.5 on $z_{\star}$ allows us to encompass the range of allowed $z_{\star}$ from CMB results (in particular [12, 44, 45]), we released the constraint on $z_{\star}$, allowing for a wider variation range, to study the impact on the warm dark matter mass bound. 

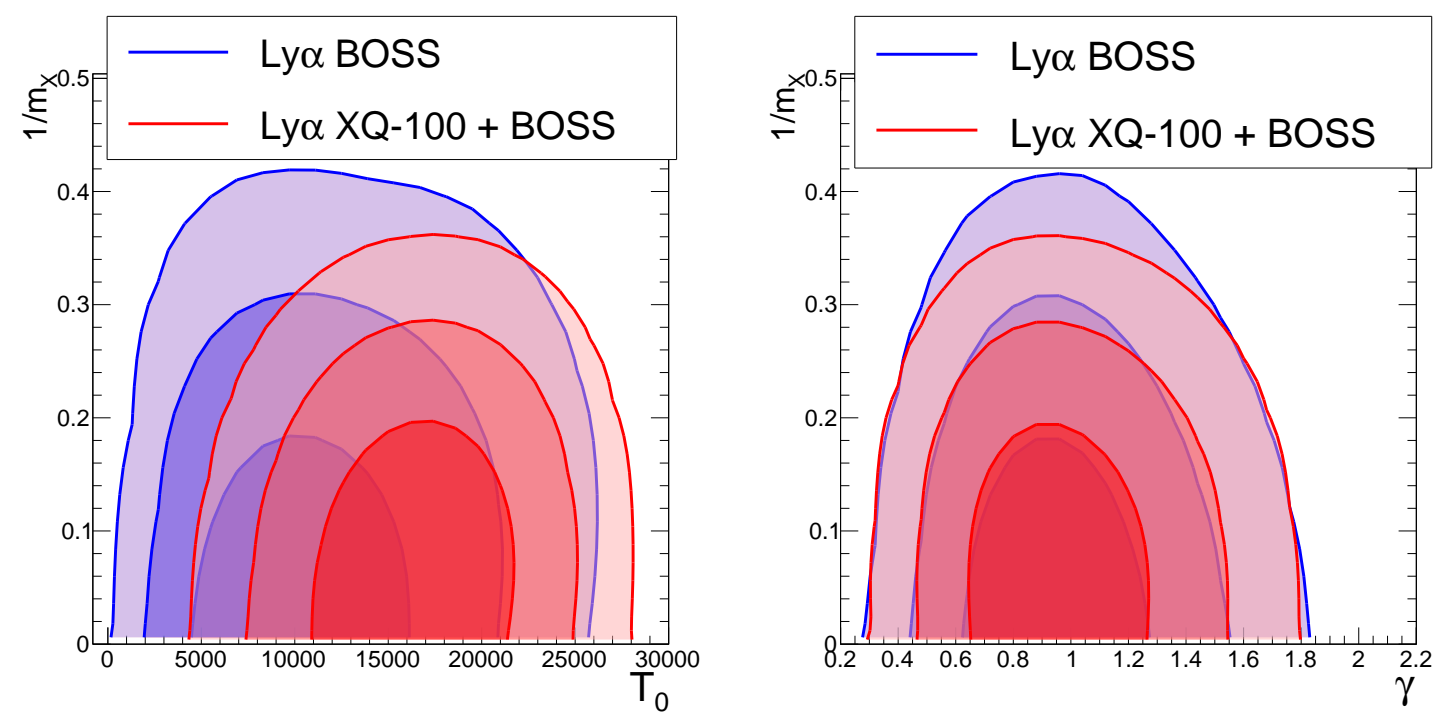

Figure 12: $2 D$ confidence level contours between IGM temperature parameters and $1 / m_{x}$. The $68 \%, 95 \%$ and $99.7 \%$ confidence contours are obtained for the BOSS Ly $\alpha$ data alone and for the combination of XQ-100 and BOSS. Left plot : $\left(T_{0}, 1 / m_{x}\right)$. Right plot: $\left(\gamma, 1 / m_{x}\right)$.

The effect is small: If we increase the sigma to 2.5 , the $95 \%$ CL limit on $m_{X}$ decreases from $4.17 \mathrm{keV}$ to $3.90 \mathrm{keV}$.

Finally, it has been recently argued in [47] that the small-scale cutoff in the power spectrum can be accounted for by a warm IGM rather than a warm DM particle. By adding XQ-100 to BOSS data, in Fig 12, we do not observe any significant change in the IGM temperature $T_{0}$ nor in the logarithmic slope $\gamma$ of the dependence of the IGM temperature with overdensity $\delta$. Additional Ly $\alpha$ forest data at higher redshifts $(z \geq 4.5)$ are needed to better study this hypothesis.

In summary, with the inclusion of XQ-100 in addition to BOSS into the analysis of BP16, we confirm our original limit from BOSS alone, in the case of $\Lambda \mathrm{WDM}$ model. We issue tighter bounds on pure dark matter particles: $m_{X} \gtrsim 4.17 \mathrm{keV}$ (95\% C.L.) for early decoupled thermal relics and its corresponding bound for a non-resonantly produced right-handed neutrino $m_{s} \gtrsim 25.0 \mathrm{keV}$ (95\% C.L.).

\subsection{Comparison with Irsic et al. Ly $\alpha$ power spectrum}

Recently, the XQ-100 team released the measurement of the Ly $\alpha$ forest power spectrum in [2]. Applying the methodology of BP16 to their XQ-100 power spectrum alone, we find a bound at $m_{X}>$ $1.90 \mathrm{keV}$ (95\% C.L.) for thermal relics, which is fully compatible with our bound, $m_{X}>2.08 \mathrm{keV}$ (95\% C.L.). We suspect that the slightly tighter limit we obtain comes from the better estimation of the resolution as explained in Sec. 2.3. By removing the highest $k$-bins, we indeed observe a small trend that may explain partially the difference. For instance, when we do not take into account the $k$-bins between 0.06 and $0.07(\mathrm{~km} / \mathrm{s})^{-1}$ for the highest redshift bin, the limit decreases from 2.08 to $2.03 \mathrm{keV}$. Tab. 4 and Fig. 13 summarize the comparison on the $95 \%$ confidence limits obtained with the two power spectra. 

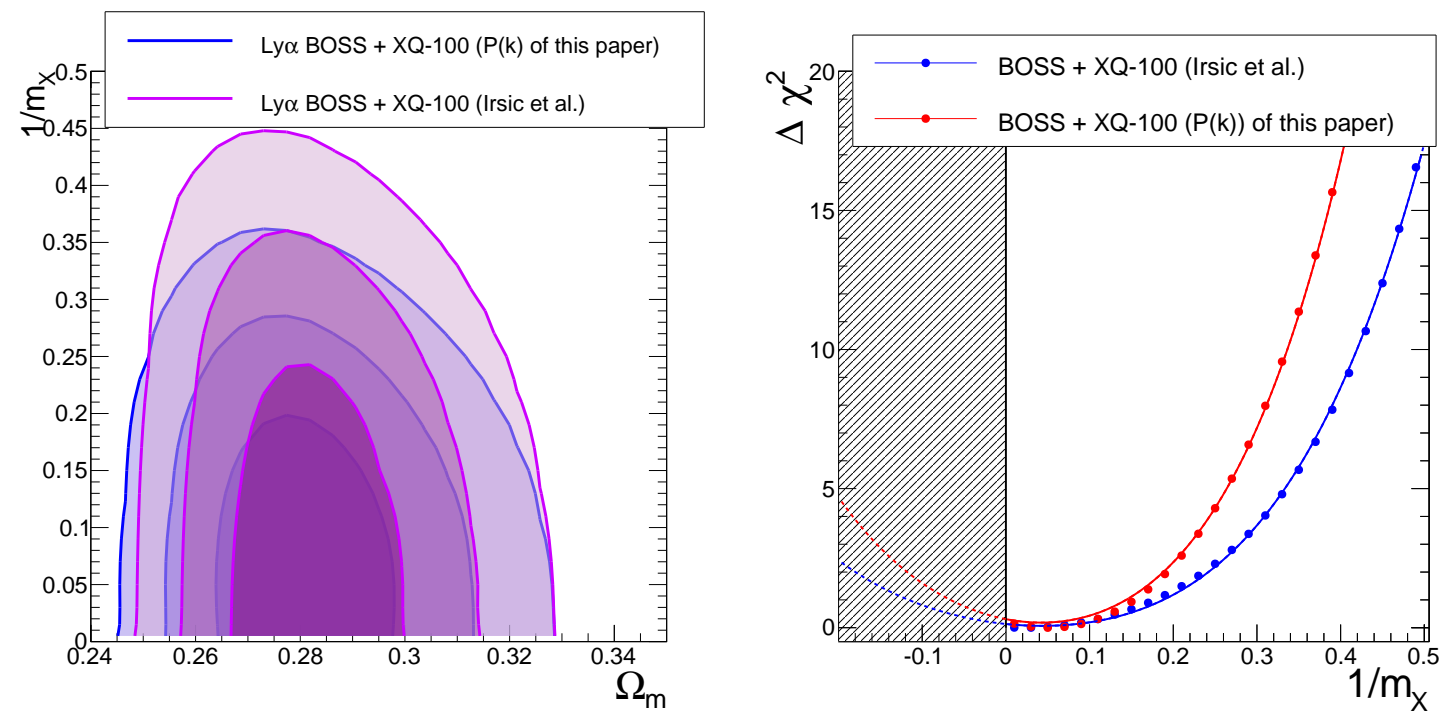

Figure 13: Comparison of the results obtained with the $P(k)$ computed in this paper and the $P(k)$ measured in [2]. Left plot : 2 D confidence level contours in the $\left(\Omega_{m}, 1 / m_{x}\right)$ plane. The $68 \%, 95 \%$ and $99.7 \%$ confidence contours are obtained for the combination of XQ-100 and BOSS data. Right plot: $\Delta \chi^{2}$ profile as a function of $\sum m_{v}$ for the combination of BOSS and XQ-100. Each point is the $\Delta \chi^{2}$ obtained after a maximization of the total likelihood over the other free parameters.

Table 4: 95\% confidence limit on $m_{X}$, the mass of thermal relics, and $m_{s}$, the mass of non-resonantly produced neutrinos, in a WDM scenario. The first row corresponds to the most stringent lower limit on WDM mass to date prior to this work, obtained with BOSS data in BY16. The other lines are for this work.

\begin{tabular}{|c|c|c|}
\hline & $P(k)$ of this paper & $P(k)$ of [2] \\
\hline BOSS alone [3] & \multicolumn{2}{|c|}{$m_{X} \gtrsim 4.09 \mathrm{keV}$ and $m_{s} \gtrsim 24.4 \mathrm{keV}$} \\
\hline XQ-100 alone & $m_{X} \gtrsim 2.08 \mathrm{keV} m_{s} \gtrsim 10.2 \mathrm{keV}$ & $m_{X} \gtrsim 1.90 \mathrm{keV} m_{s} \gtrsim 9.0 \mathrm{keV}$ \\
\hline XQ-100 + BOSS & $m_{X} \gtrsim 4.17 \mathrm{keV} m_{s} \gtrsim 25.0 \mathrm{keV}$ & $m_{X} \gtrsim 3.29 \mathrm{keV} m_{s} \gtrsim 18.4 \mathrm{keV}$ \\
\hline
\end{tabular}




\subsection{Adding HIRES/MIKE power spectrum}

The analysis presented in [48] shows that the combination of the XQ-100 and HIRES/MIKE datasets can significantly improve the limit on $m_{X}$. Indeed, the two datasets have different degeneracies between astrophysical and cosmological parameters that are disentangled when the data are combined, thanks to the higher resolution of the HIRES/MIKE spectrograph. By studying the three datasets BOSS, XQ-100 and HIRES/MIKE, we can thus expect an improvement over BOSS+XQ-100. However, as noted before, since the first snapshot of our simulations is taken at $z=4.6$, we can only use the lowest two redshift bins of HIRES/MIKE $(z=4.2$ and $z=4.6)$.
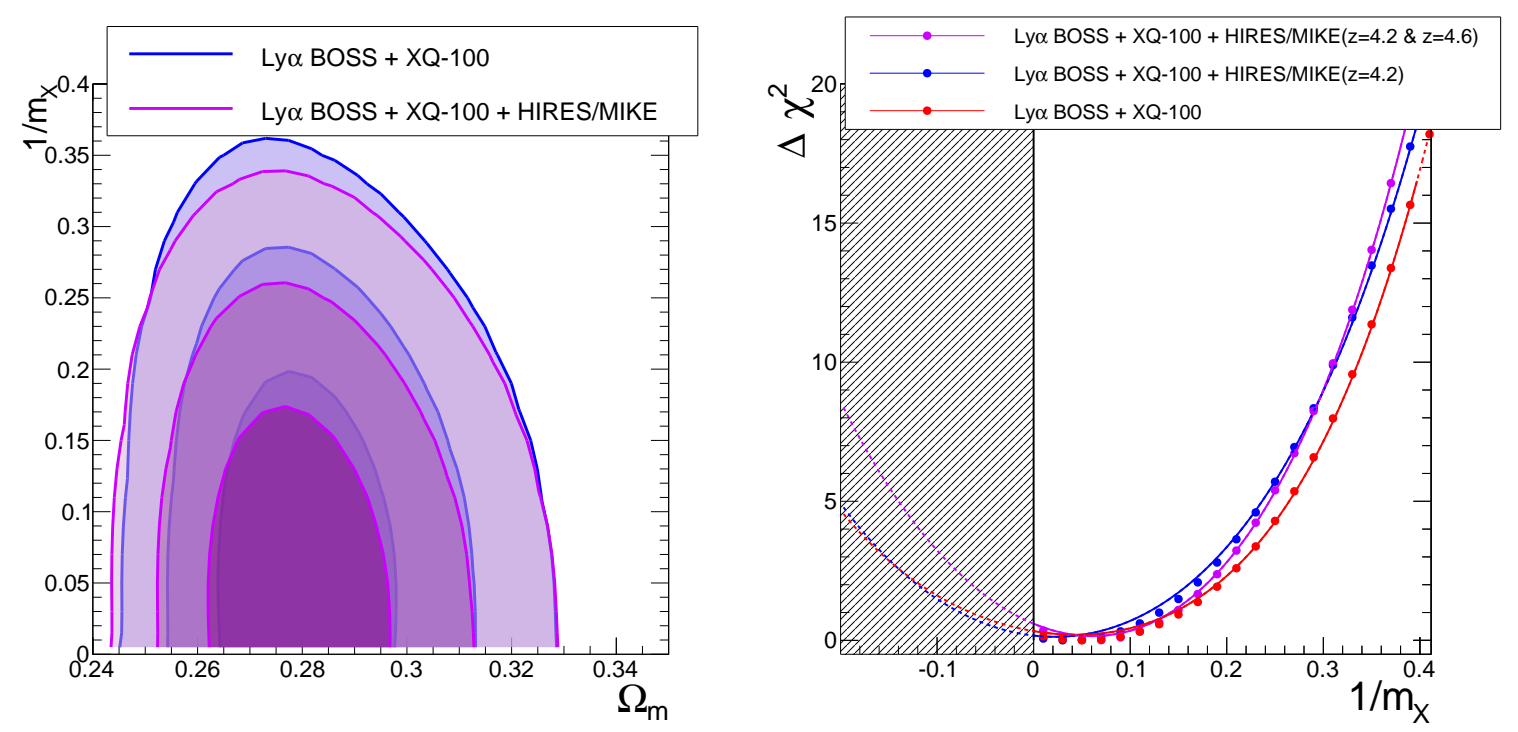

Figure 14: Left plot : $2 D$ confidence level contours for the $\left(\Omega_{m}, 1 / m_{x}\right)$ cosmological parameters. The $68 \%, 95 \%$ and $99.7 \%$ confidence contours are obtained for the combination of XQ-100 and BOSS and with adding HIRES/MIKE power spectrum. Right plot: $\Delta \chi^{2}$ profile as a function of $1 / m_{x}$ for the three configurations: the combination of (BOSS +XQ-100), (BOSS +XQ-100 + HIRES/MIKE with one redshift bin $z=4.2)$ and $(B O S S+X Q-100+H I R E S / M I K E$ with two redshift bins( $z=4.2$ and $z=4.6)$ ). Each point is the $\Delta \chi^{2}$ obtained after a maximization of the total likelihood over the other free parameters.

Fig. 14 (left) illustrates the improvement in the $1 / m_{X}-\Omega_{m}$ plane. The new bounds with HIRES/MIKE are $m_{X} \gtrsim 4.65 \mathrm{keV}$ and $m_{s} \gtrsim 28.8 \mathrm{keV}$ (95\% C.L.). As we explained in Sec. 5.1, the fit of the $\chi^{2}$ profile shown on Fig. 14 (right) by a quadratic term demonstrates a reduction of $\sigma$ from 0.123 to 0.105 and finally 0.093 when we add successively the $z=4.2$ and $z=4.6$ redshift bins of HIRES/MIKE. It represents a gain of respectively $17 \%$ and $13 \%$ in statistical sensitivity. In total, it is a gain in statistical sensitivity of $60 \%$ with respect to BOSS alone. We do not observe such an impressive gain for the 95\% CL bound because the $\chi^{2}$ minimum moves from the negative (unphysical) region to the physical region. Our final limit on $m_{X}$ is in perfect agreement with the new bound $m_{X} \gtrsim 5.3 \mathrm{keV}$ given in [48], confirming the consistent bounds measured by the various teams $[3,4,14,48]$. 


\section{Conclusion}

We measure the 1D Ly $\alpha$ power spectrum of the 100 quasars of the XQ-100 survey, applying the method [1] developed for BOSS. The power spectrum is computed with a Fourier transform. We use the flux error measured by the official pipeline [16] to estimate the noise power spectrum. Since we aim at measuring small scales that are crucial to constrain WDM, the determination of the spectrograph resolution was subject to a special dedicated treatment. Using the raw 2D quasar spectra, we checked that for many observations, the seeing is better than the slit width. Therefore, our computation of the spectrograph resolution relies upon the measured seeing at VLT instead of upon the slit width. We measured the power spectrum for three redshift bins covering the $\mathrm{H}_{\mathrm{I}}$ absorption range $3.0<z<4.2$ and reaching $k$-modes as high as $0.070 \mathrm{~s} \mathrm{~km}^{-1}$.

Fitting Ly $\alpha$ data alone leads to cosmological parameters in excellent agreement with the values derived independently from CMB data. Combining BOSS Ly $\alpha$ and XQ-100 Ly $\alpha$, we constrain the sum of neutrino masses to $\sum m_{v}<0.8 \mathrm{eV}$ (95\% C.L.) including all identified systematic uncertainties. With the addition of CMB data, this bound is tightened to $\sum m_{v}<0.14 \mathrm{eV}$ (95\% C.L.).

With their sensitivity to small scales, Ly $\alpha$ data are ideal to constrain $\Lambda$ WDM models. Using XQ-100 alone, we issue lower bounds on pure dark matter particles: $m_{X} \gtrsim 2.08 \mathrm{keV}$ (95\% C.L.) for early decoupled thermal relics, and $m_{s} \gtrsim 10.2 \mathrm{keV}$ (95\% C.L.) for non-resonantly produced right-handed neutrinos. Combining the 1D Ly $\alpha$-forest power spectrum measured by BOSS and XQ100 , we improve the two bounds to $m_{X} \gtrsim 4.17 \mathrm{keV}$ and $m_{s} \gtrsim 25.0 \mathrm{keV}$ (95\% C.L.), slightly more constraining than what was achieved in Baur et al. 2015 [3] with BOSS data alone. The $3 \sigma$ bound shows a more significant improvement, increasing from $m_{X} \gtrsim 2.74 \mathrm{keV}$ for BOSS alone to $m_{X} \gtrsim$ $3.10 \mathrm{keV}$ for the combined BOSS+XQ-100 data set. Finally, the addition of the higher-resolution HIRES/MIKE power spectrum at redshifts $z=4.2$ and 4.6 allows us to further improve the two limits to $m_{X} \gtrsim 4.65 \mathrm{keV}$ and $m_{s} \gtrsim 28.8 \mathrm{keV}$ (95\% C.L.).

\section{Acknowledgments}

We acknowledge PRACE (Partnership for Advanced Computing in Europe) for awarding us access to resources Curie thin nodes and Curie fat nodes, based in France at TGCC, under allocation numbers 2010PA2777, 2014102371 and 2012071264. This work was also granted access to the resources of CCRT under the allocation 2013-t2013047004 made by GENCI (Grand Equipement National de Calcul Intensif). The authors thank Valentina D'Odorico, Patrick Petitjean and Pasquier Noterdaeme for useful discussions on XSHOOTER. The authors are also grateful to Vid Irsic and Matteo Viel for discussions on XQ-100 Ly $\alpha$ power spectrum and for providing HIRS/MIKE power spectrum. Finally, the authors wish to thank the organizers of the Neutral H for cosmology workshop held in Berkeley in January 2017, which triggered this work. 


\section{References}

[1] Palanque-Delabrouille, N., C. Yèche, A. Borde, et al. The one-dimensional Ly-alpha forest power spectrum from BOSS. A. E A., 559:A85, 2013. arXiv: 1306.5896.

[2] Iršič, V., M. Viel, T. A. M. Berg, et al. The Lyman-alpha forest power spectrum from the XQ-100 Legacy Survey. Monthly Notices of the Royal Astronomical Society, 2016.

[3] Baur, J., N. Palanque-Delabrouille, C. Yèche, et al. Lyman-alpha forests cool warm dark matter. Journal of Cosmology and Astroparticle Physics, 8:012, 2016. arXiv:1512 .01981.

[4] Viel, M., G. D. Becker, J. S. Bolton, et al. Warm dark matter as a solution to the small scale crisis: New constraints from high redshift Lyman- $\alpha$ forest data. Physical Review D, 88:043502, 2013. arXiv: 1306.2314.

[5] Croft, R. A. C., D. H. Weinberg, M. Bolte, et al. Toward a Precise Measurement of Matter Clustering: Ly-alpha Forest Data at Redshifts 2-4. The Astrophysical Journal, 581:20-52, 2002. arXiv:astro-ph/0012324.

[6] McDonald, P., U. Seljak, S. Burles, et al. The Ly-alpha Forest Power Spectrum from the Sloan Digital Sky Survey. The Astrophysical Journal Supplement Series, 163:80-109, 2006. arXiv: astro-ph/0405013.

[7] Slosar, A., V. Iršič, D. Kirkby, et al. Measurement of baryon acoustic oscillations in the Lyman-alpha forest fluctuations in BOSS data release 9. Journal of Cosmology and Astroparticle Physics, 2013: 026-026, 2013. arXiv: 1301.3459.

[8] Busca, N. G., T. Delubac, J. Rich, et al. Baryon acoustic oscillations in the Ly-alpha forest of BOSS quasars. Astronomy $\mathcal{E}$ Astrophysics, 552:A96, 2013. arXiv: 1211.2616.

[9] Delubac, T., J. E. Bautista, N. G. Busca, et al. Baryon acoustic oscillations in the Ly $\alpha$ forest of BOSS DR11 quasars. Astronomy $\mathcal{E}$ Astrophysics, 574:A59, 2015. arXiv: 1404 . 1801.

[10] Seljak, U., A. Slosar, and P. McDonald. Cosmological parameters from combining the Lyman- $\alpha$ forest with CMB, galaxy clustering and SN constraints. Journal of Cosmology and Astroparticle Physics, 10 : 14, 2006. arXiv: astro-ph/0604335.

[11] Lesgourgues, J. and S. Pastor. Neutrino mass from Cosmology. Adv.High Energy Phys., 2012:608515, 2012. arXiv: hep-ph/1212.6154.

[12] Planck Collaboration, P. A. R. Ade, N. Aghanim, et al. Planck 2015 results. XIII. Cosmological parameters. ArXiv:1502.01589, 2015. arXiv: 1502.01589.

[13] Viel, M., J. Lesgourgues, M. G. Haehnelt, et al. Constraining warm dark matter candidates including sterile neutrinos and light gravitinos with WMAP and the Lyman- $\alpha$ forest. Physical Review D, 71: 063534, 2005. astro-ph/0501562.

[14] Seljak, U., A. Makarov, P. McDonald, et al. Can Sterile Neutrinos Be the Dark Matter? Phys. Rev. Lett., 97:191303, 2006. astro-ph/0602430.

[15] Viel, M., G. D. Becker, J. S. Bolton, et al. How Cold Is Cold Dark Matter? Small-Scales Constraints from the Flux Power Spectrum of the High-Redshift Lyman- $\alpha$ Forest. Phys. Rev. Lett., 100:041304, 2008. arXiv:0709.0131.

[16] López, S., V. D’Odorico, S. L. Ellison, et al. XQ-100: A legacy survey of one hundred $3.5 \leq z \leq 4.5$ quasars observed with VLT/X-shooter. A. $\mathcal{E}$ A., 594:A91, 2016. arXiv: 1607.08776.

[17] Borde, A., N. Palanque-Delabrouille, G. Rossi, et al. New approach for precise computation of Lyman- $\alpha$ forest power spectrum with hydrodynamical simulations. Journal of Cosmology and Astroparticle Physics, 7:005, 2014. arXiv: 1401.6472.

[18] Rossi, G., N. Palanque-Delabrouille, A. Borde, et al. Suite of hydrodynamical simulations for the Lyman- $\alpha$ forest with massive neutrinos. $A \mathcal{F}$ A, 567:A79, 2014. arXiv: 1401.6464. 
[19] Palanque-Delabrouille, N., C. Yèche, J. Baur, et al. Neutrino masses and cosmology with Lyman-alpha forest power spectrum. Journal of Cosmology and Astroparticle Physics, 11:011, 2015.

arXiv: 1506.05976.

[20] Sánchez-Ramírez, R., S. L. Ellison, J. X. Prochaska, et al. The evolution of neutral gas in damped Lyman $\alpha$ systems from the XQ-100 survey. Monthly Notices of the Royal Astronomical Society, 456: 4488-4505, 2016. arXiv: 1511.05003.

[21] XSHOOTER: Instrument's Characteristics, https://www .eso.org/sci/facilities/paranal/instruments/xshooter/inst.html.

[22] Croft, R. A. C., D. H. Weinberg, N. Katz, et al. Recovery of the Power Spectrum of Mass Fluctuations from Observations of the Ly-alpha Forest. The Astrophysical Journal, 495:44-62, 1998. arXiv: astro-ph/9708018.

[23] Frigo, M. and S. G. Johnson. FFTW: Fastest Fourier Transform in the West. 2012. ascl: 1201.015.

[24] Ahn, C. P., R. Alexandroff, C. Allende Prieto, et al. The Ninth Data Release of the Sloan Digital Sky Survey: First Spectroscopic Data from the SDSS-III Baryon Oscillation Spectroscopic Survey. ApJS, 203:21, 2012. arXiv: 1207.7137.

[25] Dawson, K. S., D. J. Schlegel, C. P. Ahn, et al. The Baryon Oscillation Spectroscopic Survey of SDSS-III. The Astronomical Journal, 145:10, 2013. arXiv: 1208.0022.

[26] Eisenstein, D. J., D. H. Weinberg, E. Agol, et al. SDSS-III: Massive Spectroscopic Surveys of the Distant Universe, the Milky Way and Extra-Solar Planetary Systems. The Astronomical Journal, 142: 72, 2011. arXiv: $1101.1529 \mathrm{v} 1$.

[27] Gunn, J. E., W. A. Siegmund, E. J. Mannery, et al. The $2.5 \mathrm{~m}$ Telescope of the Sloan Digital Sky Survey. The Astrophysical Journal, 131:2332-2359, 2006. astro-ph/0602326.

[28] Ross, N. P., A. D. Myers, E. S. Sheldon, et al. The SDSS-III Baryon Oscillation Spectroscopic Survey: Quasar Target Selection for Data Release Nine. The Astrophysical Journal Suppl., 199:3, 2012. arXiv: astro-ph.CO/1105.0606.

[29] Smee, S. A., J. E. Gunn, A. Uomoto, et al. The Multi-object, Fiber-fed Spectrographs for the Sloan Digital Sky Survey and the Baryon Oscillation Spectroscopic Survey. The Astrophysical Journal, 146: 32, 2013. arXiv: astro-ph. IM/1208.2233.

[30] Beutler, F., C. Blake, M. Colless, et al. The 6dF Galaxy Survey: baryon acoustic oscillations and the local Hubble constant. Monthly Notices of the Royal Astronomical Society, 416:3017-3032, 2011. arXiv: astro-ph.CO/1106.3366.

[31] Ross, A. J., L. Samushia, A. Burden, et al. The clustering of galaxies in the SDSS-III DR10 Baryon Oscillation Spectroscopic Survey: no detectable colour dependence of distance scale or growth rate measurements. Monthly Notices of the Royal Astronomical Society, 437:1109-1126, 2014. arXiv: astro-ph.CO/1310.1106.

[32] Anderson, L., É. Aubourg, S. Bailey, et al. The clustering of galaxies in the SDSS-III Baryon Oscillation Spectroscopic Survey: baryon acoustic oscillations in the Data Releases 10 and 11 Galaxy samples. Monthly Notices of the Royal Astronomical Society, 441:24-62, 2014. arXiv : 1312 . 4877.

[33] Springel, V., N. Yoshida, and S. D. White. GADGET: a code for collisionless and gasdynamical cosmological simulations. New Astronomy, 6:79-117, 2001. arXiv: astro-ph/0003162.

[34] Springel, V. The cosmological simulation code GADGET-2. Monthly Notices of the Royal Astronomical Society, 364:1105-1134, 2005. arXiv: astro-ph/0505010.

[35] Lewis, A., A. Challinor, and A. Lasenby. Efficient Computation of Cosmic Microwave Background Anisotropies in Closed Friedmann-Robertson-Walker Models. The Astrophysical Journal, 538: 473-476, 2000. arXiv: astro-ph/9911177. 
[36] McDonald, P. Toward a Measurement of the Cosmological Geometry at $\mathrm{z} \sim 2$ : Predicting Ly-alpha Forest Correlation in Three Dimensions and the Potential of Future Data Sets. The Astrophysical Journal, 585:34-51, 2003. arXiv: astro-ph/0108064.

[37] Planck Collaboration, P. A. R. Ade, N. Aghanim, et al. Planck 2013 results. XVI. Cosmological parameters. 2013. arXiv: 1303.5076.

[38] Meiksin, A. A. The physics of the intergalactic medium. Reviews of Modern Physics, 81:1405-1469, 2009. arXiv: 0711. 3358.

[39] Theuns, T. Numerical simulations of quasar absorbers. In Williams, P., C.-G. Shu, and B. Menard, editors, IAU Colloq. 199: Probing Galaxies through Quasar Absorption Lines, pages 185-204, 2005, astro-ph/0507570.

[40] Palanque-Delabrouille, N., C. Yèche, J. Lesgourgues, et al. Constraint on neutrino masses from SDSS-III/BOSS Ly $\alpha$ forest and other cosmological probes. Journal of Cosmology and Astroparticle Physics, 2:45, 2015. arXiv: 1410.7244.

[41] Planck Collaboration, P. A. R. Ade, N. Aghanim, et al. Planck intermediate results. XVI. Profile likelihoods for cosmological parameters. Astronomy $\mathcal{F}$ Astrophysics, 566:A54, 2014. arXiv: 1311.1657 .

[42] Gnedin, N. Y. and L. Hui. Probing the Universe with the Ly $\alpha$ forest - I. Hydrodynamics of the low-density intergalactic medium. Monthly Notices of the Royal Astronomical Society, 296:44-55, 1998. astro-ph/9706219.

[43] McDonald, P., U. Seljak, R. Cen, et al. The Linear Theory Power Spectrum from the Ly-alpha Forest in the Sloan Digital Sky Survey. The Astrophysical Journal, 635:761-783, 2005. arXiv: astro-ph/0407377.

[44] Hinshaw, G., D. Larson, E. Komatsu, et al. Nine-year Wilkinson Microwave Anisotropy Probe (WMAP) Observations: Cosmological Parameter Results. The Astrophysical Journal Supplement Series, 208:19, 2013. arXiv: astro-ph.C0/1212.5226.

[45] Planck Collaboration, N. Aghanim, M. Ashdown, et al. Planck intermediate results. XLVI. Reduction of large-scale systematic effects in HFI polarization maps and estimation of the reionization optical depth. ArXiv e-prints, 2016. arXiv: 1605.02985.

[46] Dodelson, S. and L. M. Widrow. Sterile neutrinos as dark matter. Phys. Rev. Lett., 72:17-20, 1994. hep-ph/9303287.

[47] Garzilli, A., A. Boyarsky, and O. Ruchayskiy. Cutoff in the Lyman $\{\backslash$ alpha $\}$ forest power spectrum: warm IGM or warm dark matter? ArXiv e-prints, 2015. arXiv: 1510.07006.

[48] Iršič, V., M. Viel, M. G. Haehnelt, et al. New Constraints on the free-streaming of warm dark matter from intermediate and small scale Lyman- $\alpha$ forest data. ArXiv e-prints, 2017. arXiv: 1702 .01764.

\section{Appendix: Measured XQ-100 Ly $\alpha$ flux power spectrum}

Table 5 gives the values of the power spectra from XQ-100 quasar data presented in this work. The method used to estimate the spectrograph resolution from the seeing of the observations, and the noise power spectrum from the pipeline pixel uncertainties, are described in section 2.3 . The columns list the redshift, the $k$ mode, the flux power spectrum $P_{1 D}(k)$, the statistical uncertainty $\sigma_{\text {stat }}$ and the noise power spectrum $P^{n o i s e}(k)$. The $1 \mathrm{D}$ power spectrum, $P_{1 D}(k)$ is computed as explain in Eq. 2.3, in particular the uncorrelated component related to metal absorption is subtracted.

Table 5: 1D Flux power spectrum from the XQ-100 survey, for the three redshift bins of this work. 


\begin{tabular}{|c|c|c|c|c|}
\hline$z$ & $k\left[\mathrm{~s} \mathrm{~km}^{-1}\right]$ & $P_{1 D}(z, k)\left[\mathrm{km} \mathrm{s}^{-1}\right]$ & $\sigma_{\text {stat }}(z, k)\left[\mathrm{km} \mathrm{s}^{-1}\right]$ & $P^{\text {noise }}(z, k)\left[\mathrm{km} \mathrm{s}^{-1}\right]$ \\
\hline 3.200000 & 0.001500 & 58.113500 & 4.643160 & 0.025454 \\
\hline 3.200000 & 0.002500 & 54.555800 & 3.931500 & 0.024762 \\
\hline 3.200000 & 0.003500 & 44.943600 & 3.079560 & 0.023012 \\
\hline 3.200000 & 0.004500 & 42.804400 & 3.401900 & 0.024050 \\
\hline 3.200000 & 0.005500 & 38.448600 & 3.022150 & 0.022354 \\
\hline 3.200000 & 0.006500 & 32.178500 & 2.667050 & 0.024910 \\
\hline 3.200000 & 0.007500 & 30.951600 & 2.094940 & 0.023047 \\
\hline 3.200000 & 0.008500 & 30.244900 & 2.215940 & 0.025844 \\
\hline 3.200000 & 0.009500 & 28.993000 & 2.180230 & 0.024560 \\
\hline 3.200000 & 0.010500 & 25.604600 & 1.596620 & 0.024416 \\
\hline 3.200000 & 0.011500 & 22.290900 & 1.532780 & 0.025576 \\
\hline 3.200000 & 0.012500 & 24.299100 & 1.857580 & 0.024284 \\
\hline 3.200000 & 0.013500 & 19.278000 & 1.491770 & 0.025975 \\
\hline 3.200000 & 0.014500 & 18.835600 & 1.197180 & 0.027148 \\
\hline 3.200000 & 0.015500 & 16.788400 & 1.272570 & 0.027732 \\
\hline 3.200000 & 0.016500 & 19.063900 & 1.502010 & 0.026140 \\
\hline 3.200000 & 0.017500 & 17.625900 & 1.351370 & 0.026887 \\
\hline 3.200000 & 0.018500 & 14.538700 & 0.993207 & 0.027246 \\
\hline 3.200000 & 0.019500 & 16.092300 & 1.105720 & 0.028970 \\
\hline 3.200000 & 0.020500 & 14.805700 & 1.084320 & 0.027306 \\
\hline 3.200000 & 0.021500 & 15.687800 & 1.061050 & 0.026487 \\
\hline 3.200000 & 0.022500 & 12.796500 & 0.795661 & 0.028633 \\
\hline 3.200000 & 0.023500 & 15.005400 & 1.464770 & 0.030546 \\
\hline 3.200000 & 0.024500 & 13.566500 & 1.060040 & 0.030663 \\
\hline 3.200000 & 0.025500 & 13.100100 & 1.069790 & 0.030242 \\
\hline 3.200000 & 0.026500 & 10.965000 & 0.839604 & 0.031844 \\
\hline 3.200000 & 0.027500 & 9.597180 & 0.781718 & 0.032698 \\
\hline 3.200000 & 0.028500 & 10.595200 & 0.961049 & 0.032106 \\
\hline 3.200000 & 0.029500 & 9.824680 & 0.723203 & 0.034216 \\
\hline 3.200000 & 0.030500 & 9.827700 & 0.833807 & 0.037026 \\
\hline 3.200000 & 0.031500 & 9.041100 & 0.688367 & 0.035163 \\
\hline 3.200000 & 0.032500 & 8.845860 & 0.723757 & 0.036374 \\
\hline 3.200000 & 0.033500 & 8.703450 & 0.767202 & 0.036836 \\
\hline 3.200000 & 0.034500 & 8.763500 & 0.695326 & 0.041306 \\
\hline 3.200000 & 0.035500 & 7.480200 & 0.660709 & 0.041548 \\
\hline 3.200000 & 0.036500 & 8.022700 & 0.640697 & 0.038998 \\
\hline 3.200000 & 0.037500 & 7.967960 & 0.760975 & 0.043356 \\
\hline 3.200000 & 0.038500 & 7.814740 & 0.541527 & 0.040360 \\
\hline 3.200000 & 0.039500 & 7.582290 & 0.656729 & 0.043170 \\
\hline 3.200000 & 0.040500 & 6.143250 & 0.401928 & 0.044032 \\
\hline 3.200000 & 0.041500 & 5.263360 & 0.366231 & 0.048178 \\
\hline 3.200000 & 0.042500 & 6.010660 & 0.528384 & 0.051034 \\
\hline 3.200000 & 0.043500 & 5.254620 & 0.340819 & 0.047227 \\
\hline 3.200000 & 0.044500 & 5.063790 & 0.392426 & 0.051594 \\
\hline 3.200000 & 0.045500 & 5.343530 & 0.408905 & 0.057472 \\
\hline 3.200000 & 0.046500 & 5.146310 & 0.405373 & 0.055439 \\
\hline 3.200000 & 0.047500 & 4.945440 & 0.353022 & 0.060523 \\
\hline 3.200000 & 0.048500 & 4.670380 & 0.342687 & 0.063460 \\
\hline 3.200000 & 0.049500 & 5.446050 & 0.576584 & 0.061197 \\
\hline 3.200000 & 0.050500 & 4.889070 & 0.436336 & 0.068134 \\
\hline 3.200000 & 0.051500 & 3.878910 & 0.304877 & 0.068175 \\
\hline 3.200000 & 0.052500 & 3.709630 & 0.333078 & 0.074124 \\
\hline 3.200000 & 0.053500 & 3.853920 & 0.358999 & 0.080345 \\
\hline 3.200000 & 0.054500 & 3.654200 & 0.277982 & 0.074647 \\
\hline 3.200000 & 0.055500 & 3.731370 & 0.342549 & 0.084883 \\
\hline 3.200000 & 0.056500 & 3.197610 & 0.267283 & 0.091170 \\
\hline 3.200000 & 0.057500 & 3.677780 & 0.340790 & 0.097883 \\
\hline 3.200000 & 0.058500 & 2.982880 & 0.233127 & 0.094032 \\
\hline 3.200000 & 0.059500 & 2.565260 & 0.206981 & 0.104943 \\
\hline 3.200000 & 0.060500 & 2.700700 & 0.232480 & 0.113155 \\
\hline 3.200000 & 0.061500 & 3.348200 & 0.295818 & 0.111648 \\
\hline 3.200000 & 0.062500 & 2.276380 & 0.228332 & 0.129952 \\
\hline 3.200000 & 0.063500 & 2.984240 & 0.281502 & 0.138266 \\
\hline 3.200000 & 0.064500 & 2.545250 & 0.284807 & 0.141217 \\
\hline 3.200000 & 0.065500 & 2.194860 & 0.194749 & 0.145616 \\
\hline 3.200000 & 0.066500 & 2.753030 & 0.259098 & 0.149937 \\
\hline 3.200000 & 0.067500 & 2.425180 & 0.280530 & 0.165001 \\
\hline 3.200000 & 0.068500 & 2.973450 & 0.381149 & 0.194633 \\
\hline 3.200000 & 0.069500 & 1.674630 & 0.171965 & 0.184622 \\
\hline
\end{tabular}




\begin{tabular}{|c|c|c|c|c|}
\hline$z$ & $k\left[\mathrm{~s} \mathrm{~km}^{-1}\right]$ & $P_{1 D}(z, k)\left[\mathrm{km} \mathrm{s}^{-1}\right]$ & $\sigma_{\text {stat }}(z, k)\left[\mathrm{km} \mathrm{s}^{-1}\right]$ & $P^{\text {noise }}(z, k)\left[\mathrm{km} \mathrm{s}^{-1}\right]$ \\
\hline 3.555000 & 0.001500 & 88.865100 & 6.515710 & 0.068276 \\
\hline 3.555000 & 0.002500 & 73.205800 & 5.585860 & 0.065727 \\
\hline 3.555000 & 0.003500 & 56.506800 & 4.059340 & 0.059281 \\
\hline 3.555000 & 0.004500 & 55.431500 & 4.706380 & 0.065773 \\
\hline 3.555000 & 0.005500 & 53.716600 & 3.723840 & 0.067768 \\
\hline 3.555000 & 0.006500 & 49.200200 & 4.171520 & 0.062244 \\
\hline 3.555000 & 0.007500 & 40.856000 & 2.989860 & 0.063620 \\
\hline 3.555000 & 0.008500 & 44.584000 & 3.431320 & 0.067218 \\
\hline 3.555000 & 0.009500 & 39.392600 & 2.903970 & 0.070289 \\
\hline 3.555000 & 0.010500 & 39.809200 & 3.131610 & 0.062548 \\
\hline 3.555000 & 0.011500 & 39.328800 & 3.592160 & 0.070704 \\
\hline 3.555000 & 0.012500 & 35.795000 & 2.874880 & 0.074327 \\
\hline 3.555000 & 0.013500 & 32.274600 & 2.456710 & 0.069139 \\
\hline 3.555000 & 0.014500 & 27.916700 & 1.928550 & 0.065362 \\
\hline 3.555000 & 0.015500 & 23.825100 & 1.920700 & 0.066460 \\
\hline 3.555000 & 0.016500 & 26.716800 & 2.137040 & 0.066389 \\
\hline 3.555000 & 0.017500 & 27.938800 & 2.204940 & 0.068883 \\
\hline 3.555000 & 0.018500 & 23.226400 & 1.576110 & 0.063949 \\
\hline 3.555000 & 0.019500 & 22.242000 & 1.652990 & 0.075736 \\
\hline 3.555000 & 0.020500 & 21.346200 & 1.681510 & 0.070140 \\
\hline 3.555000 & 0.021500 & 19.947400 & 1.620770 & 0.067363 \\
\hline 3.555000 & 0.022500 & 17.548600 & 1.215020 & 0.067391 \\
\hline 3.555000 & 0.023500 & 19.394300 & 1.439100 & 0.081993 \\
\hline 3.555000 & 0.024500 & 21.075800 & 1.770870 & 0.077001 \\
\hline 3.555000 & 0.025500 & 15.565100 & 1.461320 & 0.073399 \\
\hline 3.555000 & 0.026500 & 15.723200 & 1.105650 & 0.080061 \\
\hline 3.555000 & 0.027500 & 14.708600 & 1.155350 & 0.081257 \\
\hline 3.555000 & 0.028500 & 16.462900 & 1.432750 & 0.079846 \\
\hline 3.555000 & 0.029500 & 15.304700 & 1.261340 & 0.082697 \\
\hline 3.555000 & 0.030500 & 14.726000 & 1.016610 & 0.079283 \\
\hline 3.555000 & 0.031500 & 12.821200 & 1.061250 & 0.081678 \\
\hline 3.555000 & 0.032500 & 11.147600 & 0.855343 & 0.081194 \\
\hline 3.555000 & 0.033500 & 11.369800 & 0.947062 & 0.095574 \\
\hline 3.555000 & 0.034500 & 12.098200 & 0.959637 & 0.092569 \\
\hline 3.555000 & 0.035500 & 11.719500 & 0.915917 & 0.099837 \\
\hline 3.555000 & 0.036500 & 10.815400 & 0.776541 & 0.089857 \\
\hline 3.555000 & 0.037500 & 11.036900 & 0.844236 & 0.096920 \\
\hline 3.555000 & 0.038500 & 9.943440 & 0.820234 & 0.093338 \\
\hline 3.555000 & 0.039500 & 8.680970 & 0.709705 & 0.102320 \\
\hline 3.555000 & 0.040500 & 9.349400 & 0.737119 & 0.093777 \\
\hline 3.555000 & 0.041500 & 8.327400 & 0.612365 & 0.109957 \\
\hline 3.555000 & 0.042500 & 8.616410 & 0.729467 & 0.115910 \\
\hline 3.555000 & 0.043500 & 8.643760 & 0.759264 & 0.108632 \\
\hline 3.555000 & 0.044500 & 8.159150 & 0.595760 & 0.112995 \\
\hline 3.555000 & 0.045500 & 8.031460 & 0.575558 & 0.124681 \\
\hline 3.555000 & 0.046500 & 9.380490 & 0.772225 & 0.121156 \\
\hline 3.555000 & 0.047500 & 7.350790 & 0.568898 & 0.120322 \\
\hline 3.555000 & 0.048500 & 6.265940 & 0.496989 & 0.128234 \\
\hline 3.555000 & 0.049500 & 6.701070 & 0.475256 & 0.128688 \\
\hline 3.555000 & 0.050500 & 6.040380 & 0.457834 & 0.142447 \\
\hline 3.555000 & 0.051500 & 7.350400 & 0.607125 & 0.140783 \\
\hline 3.555000 & 0.052500 & 6.035630 & 0.508089 & 0.154362 \\
\hline 3.555000 & 0.053500 & 6.873980 & 0.558892 & 0.148096 \\
\hline 3.555000 & 0.054500 & 5.963720 & 0.451035 & 0.145561 \\
\hline 3.555000 & 0.055500 & 5.283380 & 0.442484 & 0.179433 \\
\hline 3.555000 & 0.056500 & 6.385500 & 0.672334 & 0.171652 \\
\hline 3.555000 & 0.057500 & 4.891520 & 0.500542 & 0.189406 \\
\hline 3.555000 & 0.058500 & 4.792700 & 0.408010 & 0.187159 \\
\hline 3.555000 & 0.059500 & 5.216490 & 0.583486 & 0.197799 \\
\hline 3.555000 & 0.060500 & 4.631330 & 0.509557 & 0.209080 \\
\hline 3.555000 & 0.061500 & 4.726460 & 0.451763 & 0.201643 \\
\hline 3.555000 & 0.062500 & 3.939570 & 0.449889 & 0.235744 \\
\hline 3.555000 & 0.063500 & 4.849110 & 0.508501 & 0.215283 \\
\hline 3.555000 & 0.064500 & 4.247100 & 0.536781 & 0.236235 \\
\hline 3.555000 & 0.065500 & 4.129100 & 0.409987 & 0.254957 \\
\hline 3.555000 & 0.066500 & 3.984800 & 0.551231 & 0.260904 \\
\hline 3.555000 & 0.067500 & 3.895950 & 0.474554 & 0.315798 \\
\hline 3.555000 & 0.068500 & 3.546240 & 0.398746 & 0.299962 \\
\hline 3.555000 & 0.069500 & 3.584180 & 0.400489 & 0.296902 \\
\hline
\end{tabular}




\begin{tabular}{|c|c|c|c|c|}
\hline$z$ & $k\left[\mathrm{~s} \mathrm{~km}^{-1}\right]$ & $P_{1 D}(z, k)\left[\mathrm{km} \mathrm{s}^{-1}\right]$ & $\sigma_{\text {stat }}(z, k)\left[\mathrm{km} \mathrm{s}^{-1}\right]$ & $P^{\text {noise }}(z, k)\left[\mathrm{km} \mathrm{s}^{-1}\right]$ \\
\hline 3.925000 & 0.001500 & 133.465000 & 10.212600 & 0.084319 \\
\hline 3.925000 & 0.002500 & 100.990000 & 6.886760 & 0.090986 \\
\hline 3.925000 & 0.003500 & 91.277800 & 5.020310 & 0.079877 \\
\hline 3.925000 & 0.004500 & 84.812100 & 6.253830 & 0.094176 \\
\hline 3.925000 & 0.005500 & 67.483600 & 4.582350 & 0.086142 \\
\hline 3.925000 & 0.006500 & 69.705300 & 4.834860 & 0.088004 \\
\hline 3.925000 & 0.007500 & 66.546700 & 4.333430 & 0.084770 \\
\hline 3.925000 & 0.008500 & 55.040100 & 5.287900 & 0.084694 \\
\hline 3.925000 & 0.009500 & 52.586500 & 3.739190 & 0.084841 \\
\hline 3.925000 & 0.010500 & 46.361200 & 2.959380 & 0.081574 \\
\hline 3.925000 & 0.011500 & 42.759000 & 2.978470 & 0.093503 \\
\hline 3.925000 & 0.012500 & 44.310500 & 3.276610 & 0.085221 \\
\hline 3.925000 & 0.013500 & 40.173000 & 2.449020 & 0.097450 \\
\hline 3.925000 & 0.014500 & 41.588900 & 2.872700 & 0.082236 \\
\hline 3.925000 & 0.015500 & 41.695000 & 2.685520 & 0.088972 \\
\hline 3.925000 & 0.016500 & 38.910000 & 2.747650 & 0.084902 \\
\hline 3.925000 & 0.017500 & 31.174100 & 2.605840 & 0.094354 \\
\hline 3.925000 & 0.018500 & 31.451900 & 2.136030 & 0.089190 \\
\hline 3.925000 & 0.019500 & 31.084700 & 2.246470 & 0.090757 \\
\hline 3.925000 & 0.020500 & 28.402200 & 2.053140 & 0.096776 \\
\hline 3.925000 & 0.021500 & 33.085800 & 2.453990 & 0.086740 \\
\hline 3.925000 & 0.022500 & 24.056600 & 1.517200 & 0.099324 \\
\hline 3.925000 & 0.023500 & 26.755200 & 2.163250 & 0.092142 \\
\hline 3.925000 & 0.024500 & 29.758100 & 2.442720 & 0.103604 \\
\hline 3.925000 & 0.025500 & 23.258200 & 1.831560 & 0.092181 \\
\hline 3.925000 & 0.026500 & 21.466500 & 1.531250 & 0.094768 \\
\hline 3.925000 & 0.027500 & 25.425000 & 2.045770 & 0.101195 \\
\hline 3.925000 & 0.028500 & 20.605800 & 1.505640 & 0.096641 \\
\hline 3.925000 & 0.029500 & 22.769000 & 1.629400 & 0.095187 \\
\hline 3.925000 & 0.030500 & 17.080000 & 1.134630 & 0.102865 \\
\hline 3.925000 & 0.031500 & 19.450900 & 1.575840 & 0.105337 \\
\hline 3.925000 & 0.032500 & 17.937600 & 1.337740 & 0.094964 \\
\hline 3.925000 & 0.033500 & 16.596200 & 1.281680 & 0.111852 \\
\hline 3.925000 & 0.034500 & 14.830300 & 1.034130 & 0.103423 \\
\hline 3.925000 & 0.035500 & 15.013800 & 1.149150 & 0.101046 \\
\hline 3.925000 & 0.036500 & 12.979600 & 0.865509 & 0.099199 \\
\hline 3.925000 & 0.037500 & 15.232900 & 1.215290 & 0.110191 \\
\hline 3.925000 & 0.038500 & 13.161600 & 1.025750 & 0.117859 \\
\hline 3.925000 & 0.039500 & 15.046400 & 1.087180 & 0.109847 \\
\hline 3.925000 & 0.040500 & 12.725100 & 0.849439 & 0.110580 \\
\hline 3.925000 & 0.041500 & 13.703400 & 1.072110 & 0.111636 \\
\hline 3.925000 & 0.042500 & 11.954200 & 0.908273 & 0.114444 \\
\hline 3.925000 & 0.043500 & 12.511800 & 0.954848 & 0.108401 \\
\hline 3.925000 & 0.044500 & 11.528500 & 0.854209 & 0.121583 \\
\hline 3.925000 & 0.045500 & 11.792000 & 1.066600 & 0.115582 \\
\hline 3.925000 & 0.046500 & 10.402500 & 0.729569 & 0.111408 \\
\hline 3.925000 & 0.047500 & 9.969150 & 0.654077 & 0.119368 \\
\hline 3.925000 & 0.048500 & 10.472600 & 0.800422 & 0.125843 \\
\hline 3.925000 & 0.049500 & 11.162100 & 0.925858 & 0.135256 \\
\hline 3.925000 & 0.050500 & 8.240230 & 0.689975 & 0.113853 \\
\hline 3.925000 & 0.051500 & 8.748400 & 0.679270 & 0.136302 \\
\hline 3.925000 & 0.052500 & 8.837000 & 0.768439 & 0.136001 \\
\hline 3.925000 & 0.053500 & 7.201600 & 0.581492 & 0.138900 \\
\hline 3.925000 & 0.054500 & 8.704030 & 0.620238 & 0.133671 \\
\hline 3.925000 & 0.055500 & 7.904330 & 0.652731 & 0.136839 \\
\hline 3.925000 & 0.056500 & 6.549770 & 0.499559 & 0.147127 \\
\hline 3.925000 & 0.057500 & 7.084960 & 0.507684 & 0.139924 \\
\hline 3.925000 & 0.058500 & 8.200610 & 0.544680 & 0.141880 \\
\hline 3.925000 & 0.059500 & 7.005290 & 0.631734 & 0.152421 \\
\hline 3.925000 & 0.060500 & 5.886190 & 0.486973 & 0.162624 \\
\hline 3.925000 & 0.061500 & 6.457330 & 0.425875 & 0.142332 \\
\hline 3.925000 & 0.062500 & 5.766620 & 0.478320 & 0.166818 \\
\hline 3.925000 & 0.063500 & 6.002120 & 0.441410 & 0.162566 \\
\hline 3.925000 & 0.064500 & 6.352780 & 0.556433 & 0.160762 \\
\hline 3.925000 & 0.065500 & 4.605540 & 0.358773 & 0.169277 \\
\hline 3.925000 & 0.066500 & 5.910770 & 0.469332 & 0.172540 \\
\hline 3.925000 & 0.067500 & 5.466390 & 0.465330 & 0.188030 \\
\hline 3.925000 & 0.068500 & 4.602840 & 0.294872 & 0.167992 \\
\hline 3.925000 & 0.069500 & 4.246550 & 0.339262 & 0.194429 \\
\hline
\end{tabular}

